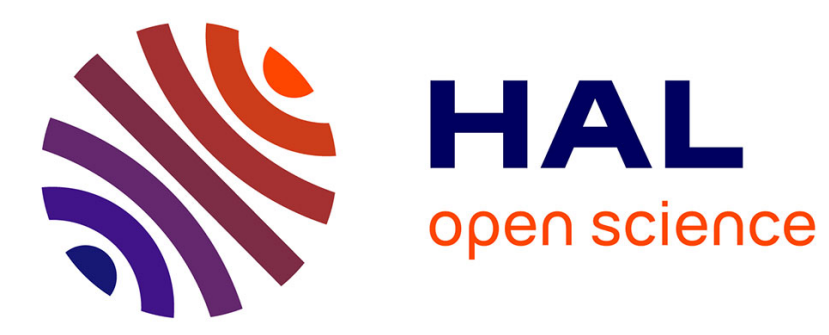

\title{
Amphores du Ve au VIIe s. à Marseille: nouvelles données sur la typologie et le contenu \\ Michel Bonifay, Dominique Pieri
}

\section{To cite this version:}

Michel Bonifay, Dominique Pieri. Amphores du Ve au VIIe s. à Marseille: nouvelles données sur la typologie et le contenu. Journal of Roman Archaeology (JRA), 1995, 8, pp.94-120. halshs-00580960

\section{HAL Id: halshs-00580960 \\ https://shs.hal.science/halshs-00580960}

Submitted on 29 Mar 2011

HAL is a multi-disciplinary open access archive for the deposit and dissemination of scientific research documents, whether they are published or not. The documents may come from teaching and research institutions in France or abroad, or from public or private research centers.
L'archive ouverte pluridisciplinaire HAL, est destinée au dépôt et à la diffusion de documents scientifiques de niveau recherche, publiés ou non, émanant des établissements d'enseignement et de recherche français ou étrangers, des laboratoires publics ou privés. 


\section{Amphores du Ve au VIIe s. à Marseille: nouvelles données sur la typologie et le contenu Michel Bonifay et Dominique Piéri}

Les fouilles archéologiques de sauvetage menées depuis plus de 25 ans à Marseille, ${ }^{1}$ ville dont $l^{\prime}$ 'importance à la fin de $l^{\prime}$ Antiquité n'est plus à démontrer, ${ }^{2}$ ont produit des masses considérables de mobiliers datant des Ve -VIIe s., dont l'étude est actuellement en cours. ${ }^{3}$

En ce qui concerne les amphores, l'examen d'une vingtaine de contextes stratigraphiques ${ }^{4}$ permet dès maintenant d'apporter de nouvelles données chronologiques et typologiques, ponctuellement enrichies par des comparaisons avec des mobiliers découverts en d'autres points du littoral français. ${ }^{5}$

Un certain nombre de ces données ont été déjà publiées plus en détail ${ }^{6}$ ou sont sur le point de l'être. ${ }^{7}$ Il nous a cependant paru utile de les regrouper ici de manière synthétique afin d'apporter une contribution au débat sur la chronologie et la diffusion des amphores tardives ouvert, dans cette collection, à propos des fouilles de Carthage. ${ }^{8}$

Nous passerons donc en revue les principales catégories d'amphores tardives présentes à Marseille: productions africaines, orientales et italiques. Ne seront pas traitées les productions hispaniques, rares à Marseille à partir du Ve s., ainsi que les productions d'origine indéterminée, souvent caractérisées par des objets incomplets. Au sein de chacune de ces productions, nous examinerons successivement la typologie, la chronologie et le contenu des principaux types d'amphores rencontrés.

\section{Remarques préliminaires}

On ne trouvera pas ici une nouvelle typologie des amphores africaines et orientales tardives, ni même un catalogue exhaustif de celles qui sont attestées à Marseille. Les quelques types

1 Les recherches d'archéologie urbaine à Marseille, inaugurées de 1967 à 1984 par le grand chantier de la Bourse (deux hectares), sont coordonnées par le Service Régional de l'Archéologie (Ministère de la Culture), avec la collaboration d'équipes diverses: l'Équipe Municipale d'Archéologie (Atelier du Patrimoine de la Ville de Marseille), le Musée d'Histoire de la Ville de Marseille, l'Association pour les Fouilles Archéologiques Nationales, le Centre Camille-Jullian et Recherches d'Antiquités Africaines (C.N.R.S.-Université de Provence, Unité Mixte de Recherche $n^{\circ}$ 9968, ex-Institut d'Archéologie Méditerranéenne).

2 R. Pernoud in Histoire du commerce de Marseille I (Paris 1949); E. Baratier, Histoire de Marseille (Toulouse 1973); S. T. Loseby, "Marseille: a late-antique success story?" JRS 82 (1992) 165-85.

3 Vaisselles de terre: M. Bonifay et al., RAN 16 (1983) 285-346; M. T. Cavaillès-Llopis, Documents d'archéologie méridionale 9 (1986) 167-95; Vaisselles de verre: D. Foy, RAN 10 (1977) 273-286; id. et M. Bonifay, RAN 17 (1984) 289-308; Amphores: M. Bonifay, RAN 19 (1986) 269-305.

4 Nous remercions les collègues qui nous ont autorisé à utiliser leur matériel: R. Boiron (AFAN, chantier du Cap Titol), A. Hesnard (CNRS, chantier de la place Jules Verne), M. Moliner (Équipe municipale d'Archéologie, chantier de la rue de la Cathédrale) et $\mathrm{P}$. Reynaud (AFAN, chantier du Bon-Jésus) à qui nous devons également le dessin des objets $\mathrm{n}^{\circ} 16,21,22,23,67$.

5 Nous remercions nos collègues du Département des Recherches Archéologiques Sous-Marines (Directeur: R. Lequément): L. Long (Conservateur du Patrimoine, épave de la Palud), M.-P. Jézégou (Ingénieur d'Etude, épave de Saint-Gervais II).

6 M. Bonifay, RAN 19 (1986) 269-305; id. et F. Villedieu, in Recherches sur la céramique byzantine (BCH Suppl. 18, 1989) 17-46;

$7 \quad$ N. Ben Lazreg et al., in Actes du Vle colloque d'histoire et d'archéologie de l'Afrique (Pau 1993) [sous presse]; D. Pieri in Etudes massaliètes 5; R. Boiron et al. in Etudes massaliètes 5 [à paraître].

$8 \quad$ R. Tomber in JRA 6 (1993) 142-66; J. Freed in Bir el Knissia at Carthage 1 (JRA Suppl.7) 73-92. 
illustrés ont été choisis en fonction de leur représentativité au sein du matériel découvert dans notre ville et des données nouvelles dont nous disposons à leur sujet du point de vue de leur chronologie et de la nature de leur contenu.

Par ailleurs nous demandons au lecteur d'accueillir nos propositions de datation avec le plus sévère esprit critique. Chacun sait en effet que les difficultés rencontrées pour l'établissement d'une chronologie absolue sont particulièrement aggravées en Méditerranée occidentale durant l'Antiquité tardive en raison de la longue durée de circulation du monnayage et de la datation encore controversée des sigillées africaines. Un tableau placé en annexe résume les propositions de datation des différents contextes étudiés et les critères qui ont servi à les établir (annexe 3).

Enfin, en ce qui concerne le contenu des amphores, il convient de signaler que notre matériel n'a fait l'objet d'aucune analyse chimique. Nous nous bornerons tout simplement à signaler si les exemplaires étudiés portent ou non les traces d'un enduit intérieur de poix. ${ }^{9}$ Le cas échéant le poissage permet d'exclure un contenu oléagineux ${ }^{10}$ mais pas de préciser la nature du produit: vin, salaisons ou autre. ${ }^{11}$

\section{PRODUCTIONS AFRICAINES}

Sauf exception, nous utilisons la typologie de S. J. Keay ${ }^{12}$ en essayant de l'intégrer à celle de C. Panella. ${ }^{13}$

\section{Conteneurs cylindriques de moyenne dimension (Type Keay XXV-2)}

Si l'on considère le type Keay XXV sous l'angle non pas de ses nombreuses variantes (de $\mathrm{A}$ à Z4) mais de ses trois principaux sous-types (sous-type $1=$ variantes $A-C$; sous-type 2 = variantes D-I; sous-type 3 = variantes J-W), on constate que le sous-type 2 , au sein duquel on est tenté de regrouper les figures Ostia IV, 146-47, se distingue nettement des deux autres: il est caractérisé par un bord à large collerette évasée, des anses étroitement collées au col qui est lui-même franchement cylindrique - et non plus tronconique - tout comme la panse et la pointe.

A Marseille, au milieu du Ve s. (contexte 1), seul ce sous-type 2 est présent (fig. $1 \mathbf{n}^{\circ} \mathbf{1}$ ), quoiqu'en faible proportion. C'est une situation bien différente de celle du IVe s., illustrée par les épaves de Pampelonne ${ }^{14}$ ou d'Héliopolis ${ }^{15}$ qui livrent seulement le type Keay XXV.1, ou encore l'épave $B$ de la Pointe de la Luque ${ }^{16}$ où les sous-types 1 et 3 sont associés. A Arles, dans un contexte du premier quart du Ve s. ${ }^{17}$ les trois sous-types sont attestés aux côtés des premiers témoins d'amphores cylindriques de grande dimension.

Les exemplaires recueillis dans le port de Marseille, comme tous ceux examinés en d'autres points du littoral français, ${ }^{18}$ sont poissés. Cela semble exclure un contenu oléagineux mais on sait

9 Dont la conservation a été rendue possible par le sédiment vaseux du port de Marseille.

10 Discussion résumée dans G. Charlin et al., Archeonautica 2 (1978) 21-22.

11 Ben Lazreg et al. (supra n.7).

12 Late Roman amphorae in the Western Mediterranean, A typology and economic study: the Catalan evidence (BAR S 196, Oxford 1984); ci-après "Keay".

13 "Le anfore africane della prima, media e tarda étà imperiale, tipologia e problemi," in Actes du colloque sur la céramique antique de Carthage (23-24 juin 1980) (CEDAC, Carthage 1982) p.171-86; ci-après "Panella".

14 R. Lequément, RAN 1976, 181.

15 J.-P. Joncheray in Bilan scientifique du Département des Recherches Archéologiques Sous-marines 1992 (Paris 1993).

16 B. Liou, Gallia 31 (1973) 584.

17 G. Congès, M. Leguilloux, RAN 24 (1991) 201 ss., fig.7 et 11.

18 Ben Lazreg et al. (supra n.7). 




Fig. 1. Amphores africaines. Type Keay XXV sous-type $2\left(\mathrm{n}^{\circ} 1\right)$, spatheia classiques $\left(\mathrm{n}^{\circ} 2-4\right)$, spatheia tardifs ( $\left.n^{\circ} 5-6\right)$, type Keay XXVII et XXXVI ( $n^{\circ} 7$ et 8$)$.

que certains exemplaires découverts sur l'épave du Dramont E à Saint-Raphaël contenaient des conserves d'olives. ${ }^{19}$

19 J.-P. Joncheray, Cahiers d'archéologie subaquatique 4 (1975) 141-46. 


\section{Spatheia}

Sous ce terme on regroupe au moins trois types distincts d'objets:

Spatheia "classiques" (type Ostia IV, fig. 162-65)

On a pris l'habitude, dans la littérature archéologique, de nommer spatheia (Panella 178) des amphores de même forme que le type Keay XXV.2 mais de dimension réduite. Cependant il est peut-être illusoire de vouloir les distinguer car, comme le montre le chargement de l'épave du Dramont E, il existe toute une gradation de tailles entre les amphores attribuées au type Keay XXV.2 et celles rangées sous le terme générique de spatheion. ${ }^{20}$

A Marseille, ces modèles réduits $\mathrm{d}$ 'amphores cylindriques de moyenne dimension sont particulièrement fréquents au milieu du Ve $\mathrm{s}$. (contexte 1). C'est également le cas à Tarragone, dans le dépotoir de Vila-Roma avec des monnaies de $440,{ }^{21}$ même si de nombreux exemplaires apparaissent déjà à Arles au début du siècle. ${ }^{22}$ On connaît alors assez bien leur typologie (fig. $1 \mathbf{n}^{\circ}$ 2 et 3 ) et on remarque qu'ils portent tous les traces d'un revêtement intérieur de poix.

\section{Autres spatheia}

Les spatheia restent nombreux dans les contextes marseillais datés de la deuxième moitié du Ve $s .{ }^{23}$ et malgré leur aspect très fragmenté ils ne paraissent pas résiduels car leur morphologie évolue vers une taille encore plus réduite et une simplification du bord (fig. $1 \mathbf{n}^{\circ} \mathbf{4}$ ). Dans les contextes 9 et 10, ces spatheia sont les seules formes d'amphores africaines clairement attestées; dans le contexte 12 , c'est également le type le mieux représenté au sein des productions africaines. Un certain nombre de tessons portent des traces de poix.

\section{Spatheia tardifs de très petite dimension (type Panella fig. 29)}

Il s'agit de très petits conteneurs, caractérisés par un bord convexe parfois facetté (fig. $1 \mathbf{n}^{\circ} \mathbf{5}$ et 6) La position stratigraphique des ces objets à Marseille n'est pas claire: ils apparaissent seulement dans des contextes postérieurs au Ve s. mais leur rareté dans les contextes de la fin du VIe ou du début du VIIe s. (contextes 2, 7,8) pourrait indiquer une date extrêmement tardive. En renfort de cette hypothèse, on remarque, ailleurs en Méditerranée, que ces spatheia de très petite dimension sont principalement attestés au VIIe s., ainsi sur l'épave de Yassi-Ada, ${ }^{24}$ à Saraçhane, ${ }^{25}$ ou encore à Benghazi. ${ }^{26} \mathrm{Il}$ en est de même des exemplaires encore rares découverts en Provence, ainsi à Saint-Blaise ${ }^{27}$ ou sur l'épave de Saint-Gervais II à Fos. ${ }^{28}$ La pâte beige poreuse de ces petits spatheia n'est pas forcément un argument pour une origine non africaine car elle ressemble à celle des séries les plus tardives de conteneurs cylindriques africains de grande dimension (types Keay LXI/LXII).

20 Ibid. 145. M. Claude Santamaria qui prépare la publication d'ensemble du gisement a bien voulu nous laisser examiner, en 1993, son matériel. Qu'il en soit ici remercié.

21 J. A. Remolà i Vallverdù et A. Abello i Riley, in TED'A, Un abocador del segle $V d$. C. en el forum provincial de Tarraco (Tarragone 1989) 258.

22 RAN 24 (1991) 216.

23 La situation est moins claire dans les contextes du VIe s.

24 G. Bass, F. Van Doornick, Yassi Ada I; a seventh century shipwreck (Texas 1982) 181.

25 J. W. Hayes, Excavation at Saraçhane II: the pottery (Princeton 1992) 67, type 14.

26 J. A. Riley, in Excavations at Sidi Khrebish-Benghazi (Berenice) II (LASuppl.1979) 226-27, fig.92, $\mathrm{n}^{\circ} 362$ et 364. Ces deux fragments sont issus de la série des contextes 147-152, en association avec des sigillées africaines de formes Hayes 91D, 105, 107, 109 et des sigillées phocéennes tardives (Late Roman C ware) de forme Hayes 10A. En revanche, le $n^{\circ} 363$ est d'un type différent: il est issu d'un contexte milieu VIe s. (contexte 142).

27 F. Villedieu, in G. Démians d'Archimbaud, L'oppidum de Saint-Blaise (Documents d'Archéologie Française 45,1994$) 135$, fig.79 n 7-10.

28 M. P. Jezegou in Études massaliètes 5. 


\section{Amphores cylindriques de grande dimension}

A l'intérieur de cette catégorie définie par Panella, il semble possible aujourd'hui de distinguer plusieurs groupes, sinon même plusieurs générations successives d'amphores.

Première génération d'amphores cylindriques de grande dimension

Types Keay XXVII et XXXVI

On a souligné à plusieurs reprises ${ }^{29}$ les affinités typologiques entre ces deux amphores, par exemple la soudure des anses immédiatement sous la lèvre, ce caractère ne se rencontrant sur aucun autre type d'amphore africaine. Toutefois leur forme générale est très différente: très haute et fusiforme pour l'une (type Keay XXVII), plus basse et plus pansue pour l'autre (type Keay XXXVI). La pâte est également assez particulière: extrêmement granuleuse elle est surtout très plastique, ce qui provoque de fréquentes bavures d'argile, notamment à l'intérieur du vase.

A Marseille, le type Keay XXVII (fig. $1 \mathbf{n}^{\circ}$ 7) est attesté au milieu du Ve s. (contexte 1 ) et le type Keay XXXVI (fig. $1 \mathbf{n}^{\circ} 8$ ) tout au long de ce siècle (contextes 1, 9, 10, 12). Ce dernier apparaît déjà au début du Ve s. à Arles $^{30}$ tandis que le type XXVII est fréquent à Carthage dans les contextes de la deuxième moitié du Ve s. et du début du VIe s. ${ }^{31}$

On remarque que ces deux types d'amphores ne sont pas systématiquement poissés. ${ }^{32}$

\section{Type Keay XXXV}

Même si les deux variantes sont associées sur l'épave du Dramont $\mathrm{E}^{33}$ seule la variante $\mathrm{B}$ est réellement fréquente à Marseille (fig. $2 \mathrm{n}^{\circ}$ 9-11). Toutefois on ne sait pas si les fragments récupérés appartiennent tous au modèle standard de cette amphore cylindrique de grande dimension (environ $1,00 \mathrm{~m}$ de haut pour $0,35 \mathrm{~m}$ de diamètre) comme sur le Dramont $\mathrm{E}^{34}$ et à Tarragone ${ }^{35}$ ou bien si certains proviennent de conteneurs de très grande dimension comme celui exposé au Musée de Carthage ${ }^{36}$ ou au contraire de dimension plus réduite comme celui exposé au Musée d'Agde. ${ }^{37}$ Une caractéristique de ce type d'amphore semble encore résider dans son fond court conique (fig. 2 $\mathbf{n}^{\circ}$ 11) portant assez souvent des graffiti tracés avant cuisson: une croix, un ou deux ' $V^{\prime}, 38$

Le type Keay XXXVB est l'amphore africaine la plus fréquente dans les contextes du milieu $\mathrm{du}$ Ve s. (contexte 1) et semble encore perdurer dans la deuxième moitié du siècle (contextes 9,10 et 12). Il n'est pas impossible qu'il y ait une évolution typologique en direction du type Keay LXXXV. Ces données sont en contradiction avec celles obtenues à Carthage ${ }^{39}$ où l'amphore Keay XXXVB paraît surtout typique du premier tiers du Ve s.

Tous les exemplaires du type Keay XXXVB découverts en France en milieu humide sont poissés, ${ }^{40}$ ce qui exclut l'huile comme contenu de cette amphore. ${ }^{41}$

Keay 240; Bonifay, RAN 19 (1986) 278; Freed (supra n. 8) 87.

RAN 24 (1991) 216.

M., G. Fulford et D. P. S. Peacock, Excavations at Carthage: The British Mission, Vol. I, 2, (Sheffield 1984)

130 et fig. $39, \mathrm{n}^{\circ}$ 53-55.

Ben Lazreg et al. (supra n.7) fig.10.

P. Pomey et al., Gallia informations 1987-88, 40.

Joncheray (supra n.19) 145.

Keay 234 et fig.30.

Nouvelle salle des amphores.

Ostia IV, pl. lxxvi, n 569.

Cf. également Remolà i Vallverdù (supra n.21) fig. $138, n^{\circ} 8.60$.

Freed (supra n.8) 87.

Ben Lazreg et al. (supra n.7).

En revanche les exemplaires de la variante A du type Keay XXXV découverts sur l'épave du Dramont E ne sont pas poissés (communication personnelle de M. Claude Santamaria). 
Typologie et contenu des amphores du Ve au VIIe s. à Marseille 99

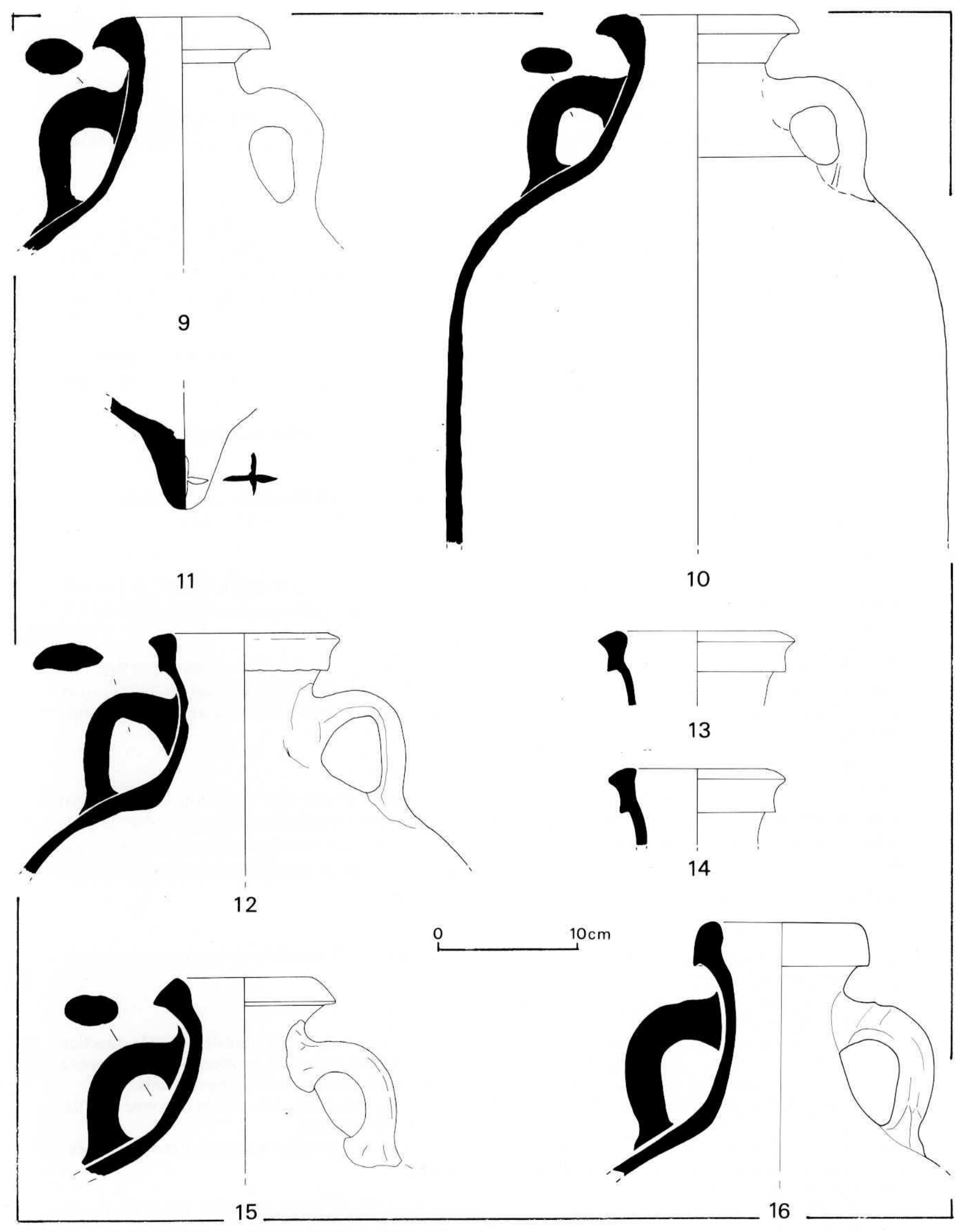

Fig. 2 Amphores africaines. Types Keay XXXVB ( $\left.{ }^{\circ} 9-11\right)$, Keay VIIIB ( $\left.{ }^{\circ} 12-14\right)$, XL-XLI ( $\left.{ }^{\circ} 15-16\right)$. 


\section{Deuxième génération d'amphores cylindriques de grande dimension}

Nous regroupons ici les types qui apparaissent à Marseille dans les contextes de la deuxième moitié du Ve s. Il est remarquable que beaucoup se trouvent associés au sein du lot d'amphores recueilli dans la maçonnerie de la coupole du baptistère d'Albenga. ${ }^{42}$

Type Keay VIIIB

L'amphore $\mathrm{n}^{\circ} 10$ du baptistère d'Albenga, ${ }^{43}$ aux côtés d'un exemplaire publié en Roumanie, ${ }^{44}$ sont parmi les rares objets complets de ce type qui reste encore mal documenté. Pourtant l'origine africaine de cette amphore ne paraît pas faire de doute si l'on en juge par son abondance en Tunisie, notamment sur le site de Yonga, au sud de Sfax, qui pourrait bien être l'un des centres producteurs ${ }^{45}$ (fig. $2 \mathbf{n}^{\circ} \mathbf{1 2}$ ).

A Marseille (fig. $2 \mathrm{n}^{\circ} 13$ et 14), cette amphore se rencontre en faible quantité dans les contextes de la fin du Ve s. et du début du VIe s. (contextes 6,10 et 12). Cette datation correspond à celle enregistrée à Carthage par la mission britannique. ${ }^{46}$

On ne dispose à Marseille d'aucune information sur le contenu de cette amphore.

Types Keay XL et XLI

Les rares fragments de cette forme découverts à Marseille proviennent de contextes de la deuxième moitié du Ves. ou du début du Vle s. (contextes 12 et 13) (fig. $2 \mathrm{n}^{\circ} 15$ et 16).

Type Keay LVII

Ce type est difficile à distinguer du type Africaine IIC de Panella (Panella p.586). Il possède cependant un bord à section plus épaisse et porte toujours une décoration peignée sur le haut du col; sa panse est parfaitement cylindrique. ${ }^{47}$

A Marseille (fig. $3 n^{\circ} 17$ et 18 ) cette amphore se rencontre de manière très sporadique dans les contextes de la fin du Ve ou du début du VIe s. (contextes 5, 6 et 12). Cette date est en accord avec celle obtenue sur le site de Carthage ${ }^{48}$ et sur celui du Nador en Algérie, où cette amphore est bien attestée dans les couches d'abandon d'époque vandale tardive..$^{49}$

\section{Type Keay LV}

Cette amphore est tout autant caractéristique par la forme de son col, entièrement cylindrique, avec un ressaut à l'intérieur, et un décor peigné à l'extérieur, que par sa pâte, le plus souvent rouge brique à surface grise. En revanche la forme du fond paraît très classique, un simple bouton parfois mouluré ${ }^{50}$ sans rapport avec les fonds à renflement annulaire des types Keay LXLXI-LXII.

42 F. Pallares, Rev.Et.Lig 53 (1987) 269-306. Nous remercions Mme C. Paoli Maineri de nous avoir donné la possibilité d'examiner ce matérel au Musée d'Albenga.

43 Ibid. fig.17.

44 A. Radulescu, Pontica 9 (1976) 99 ss., pl.VI n ${ }^{\circ} 2$.

45 Prospections conduites dans le cadre de la mission tuniso-française du littoral tunisien, sous la direction de MM. H. Slim et P. Trousset. Les observations concernant une éventuelle production d'amphores à Yonga sont dues à M. Nejib Ben Lazreg, Chercheur à l'Institut National du Patrimoine de Tunis.

46 Fulford et Peacock (supra n.31) 135 et fig.41, $n^{\circ}$ 90-91. Le fragment découvert dans le dépotoir de VilaRoma dans un contexte du second quart du Ve s. est probablement intrusif.

47 Radulescu (supra n.44) pl.V n 4. Cf. également l'exemplaire complet présenté dans le Musée Paléochrétien de Carthage (musée de la mission américaine).

48 Fulford et Peacock (supra n.31) 130 et fig.39, $n^{\circ}$ 58-60.

49 D, Manacorda in Il castellum di Nador (Monografie di Archeologia Libyca 23, 1989) 179 et fig.42, $\mathrm{n}^{\circ}$ 227-28.

50 L. Long et G. Volpe, Vetera Christianorum 31 (1994) 211 ss., fig.7, n 4-5. 
Typologie et contenu des amphores du Ve au VIIe s. à Marseille 101



17

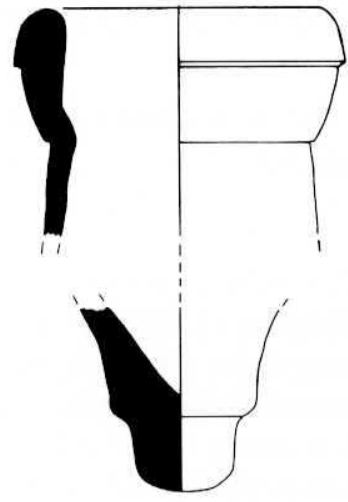

21
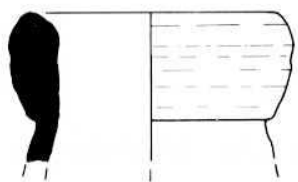

18

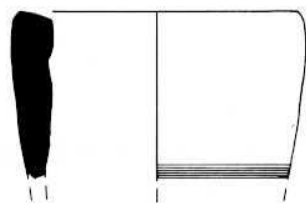

19

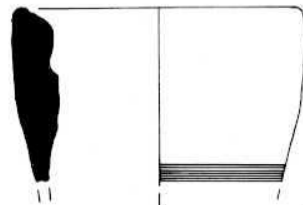

20

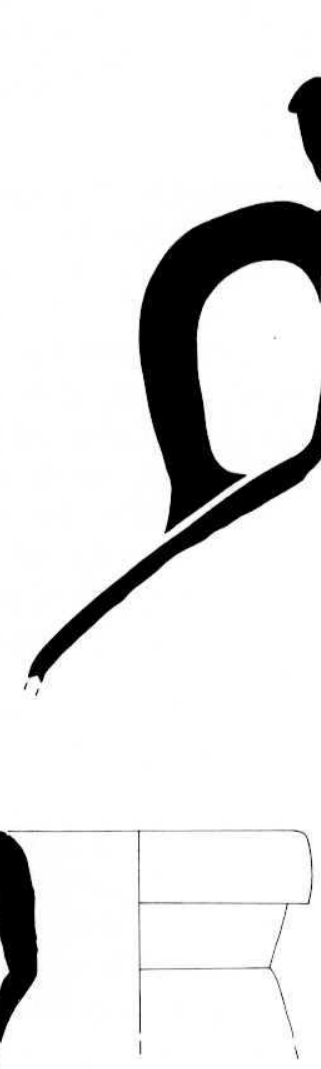

24

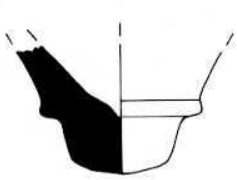

25
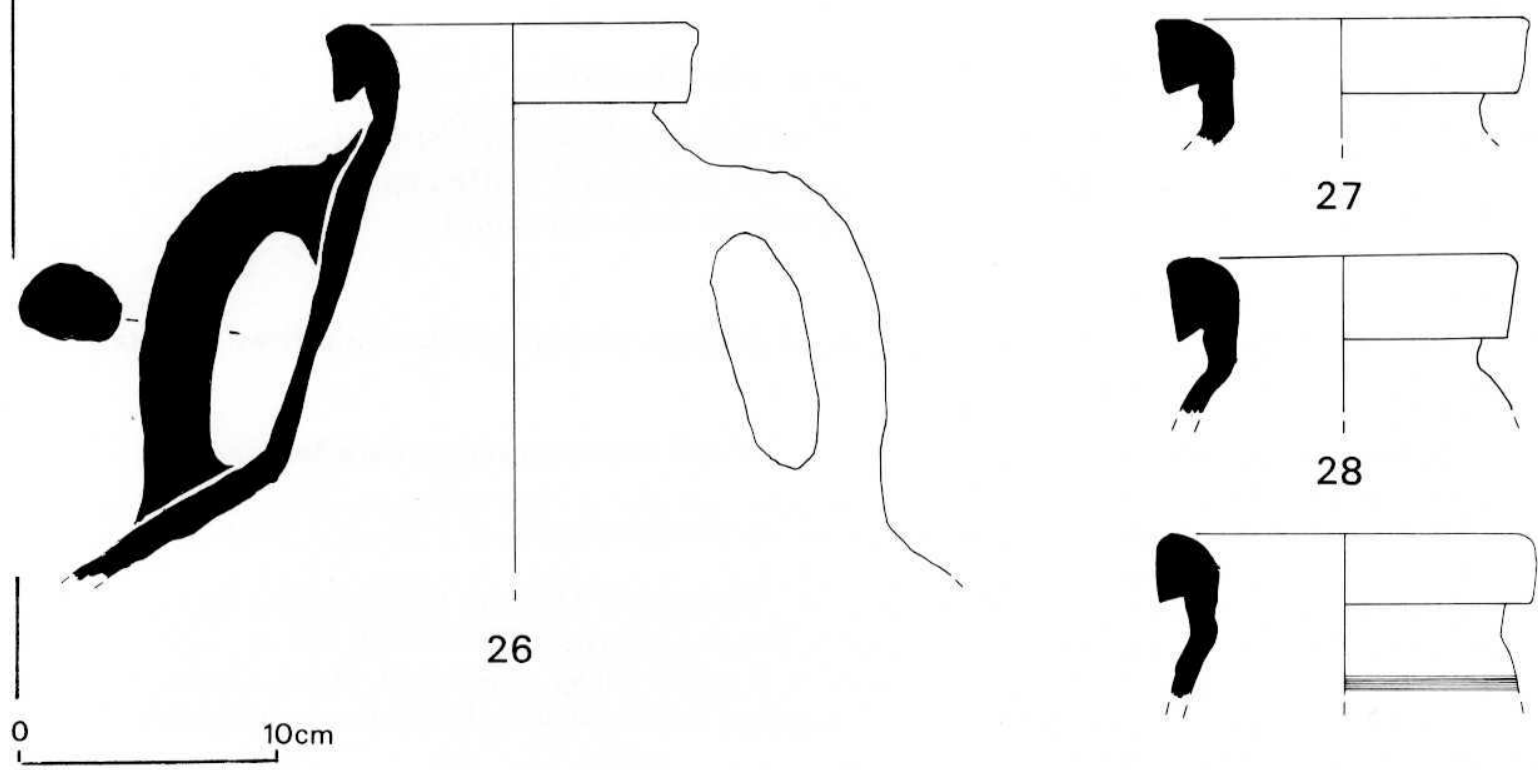

27

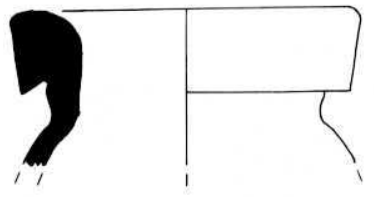

28



29

Fig. 3. Amphores africaines. Types Keay LVII ( $\left.n^{\circ} 17-18\right)$, LV ( $\left.{ }^{\circ} 19-20\right)$, Albenga 11-12 ( $\left.{ }^{\circ} 21-22\right)$, Keay LXIIA ( $\left.{ }^{\circ} 23-25\right)$, Keay LXII variante tardive ( $\left.{ }^{\circ} 26-29\right)$. 
Comme cette amphore ne s'est pas révélée, jusqu'à présent, très fréquente à Marseille (fig. $3 \mathbf{n}^{\circ}$ 19 et 20), sa position stratigraphique n'en est que moins claire. Elle semble apparaître avant le type Keay LXIIA, dans des contextes de la fin du Ve s. ou du début du VIe s. (contexte 5), mais paraît plus abondante, en association avec ce dernier type, au VIe s. (contexte 6). Le chargement de l'épave de la Palud à Port-Cros, datée du milieu du VIe s., comprend une forte proportion de ce type d'amphore, associée au type Keay LXII. ${ }^{51}$

On remarque encore que plusieurs exemplaires d'amphores Keay LV issus de sites différents ont révélé des traces de poix, ${ }^{52}$ ce qui exclut pour eux un contenu oléagineux. ${ }^{53}$

Type Albenga 11-12 (Keay LXIIQ)

Comme l'a bien montré une étude récente, ${ }^{54}$ il convient de distinguer cette amphore de la série des conteneurs regroupés par Keay sous le $n^{\circ}$ LXII: le bord de cette amphore forme un bandeau pansu, souvent concave à l'intérieur, surmonté d'une petite lèvre triangulaire débordante (fig. 3 $\mathrm{n}^{\circ} 21$ et 22). Le col, au lieu d'être tronconique, très renflé et étranglé sous le bord comme dans le type LXIIA, est ici étroit, presque cylindrique (Keay fig. $33 \mathrm{n}^{\circ} 7$ ). ${ }^{55}$ Les anses assez minces sont disposées en oreilles très larges de part et d'autre du col. Le fond pose problème: cylindrique à base aplâtie sur l'exemplaire complet $\mathrm{d}^{\prime}$ Albenga, ${ }^{56}$ il est en revanche du type cylindrique à renflement annulaire sur un exemplaire de Marseille (fig. $3 n^{\circ}$ 21); il y a peut-être là l'indice d'une évolution typologique.

La pâte des exemplaires marseillais est généralement de couleur brun-orangé, assez tendre, sableuse, avec des inclusions de quartz et de calcite.

A Marseille cette amphore précède le type Keay LXIIA dans des contextes de la deuxième moitié du Ve s. (contextes 9,10 et 12) mais se retrouve également associée à ce dernier dans des contextes du VIe s. (contexte 13). Ailleurs en Méditerranée cette amphore est généralement documentée entre la fin du Ve s. et le milieu du VIe s., par exemple à Carthage. ${ }^{57}$ Sur l'épave de la Palud à Port-Cros, ${ }^{58}$ elle est associée au type Keay LV et à différentes variantes du type Keay LXIIA.

Nous ne disposons à Marseille d'aucune information sur le contenu de cette amphore; un col récupéré à Giglio Porto est poissé. ${ }^{59}$

\section{Troisième génération d'amphores cylindriques de grande dimension}

La même complexité se retrouve dans la série des amphores Keay LX-LXI-LXII et VIIIA. Ces quatre types $\mathrm{d}^{\prime}$ amphores cylindriques ont en commun un fond à renflement annulaire très caractéristique (fig. $3 \mathbf{n}^{\circ} \mathbf{2 6}$ ), mais le détail de leur typologie reste mal connu.

Ibid. En revanche elle est absente du lot d'amphore d'Albenga, où seul le type voisin Keay LVI est représenté (Pallarès [supra n.42] 282).

52 Ben Lazreg et al. (supra n.7).

53 Il est à noter que les exemplaires recueillis sur l'épave de la Palud ne portent aucune trace de poix.

54 Boiron et al. (supra n.7).

55 Comparer avec les exemplaires de type Keay LXIIA, D et F sur la même figure.

56 Pallarès (supra n.42) fig.18-19.

57 J. W. Hayes in Excavations at Carthage conducted by the University of Michigan 1 (Tunis 1976) fig. $4 \mathrm{n}^{\circ}$ 71; C. Panella in Actes du colloque sur la céramique antique de Carthage (CEDAC 1982) 171 ss., fig.26; Fulford et Peacock (supra n.31) fig. $39, \mathrm{n}^{\circ} 64$. Le col $\mathrm{n}^{\circ} 9$ publié dans J. Freed et H. Hurst, Carthage CEDAC Bulletin 12 (juin 1991) 26, avec son col tronconique à étranglement et ses anses rapprochées, appartient plutôt au type Keay LXIIA.

58 Long et Volpe (supra n.50) fig. $6 \mathrm{n}^{\circ} 3$.

59 M. Celuzza et P. Rendini, Relitti di storia, Archeologia Subacquea in Maremma, Catalogue de l'exposition (Grosseto 13 juillet-30 septembre 1991) (Siena 1992) 114 et pl. IV nº 123. 


\section{Typologie et contenu des amphores du Ve au VIIe s. à Marseille 103}

\section{Type Keay LXII A}

C'est le seul type réellement homogène de la vaste typologie regroupée par Keay sous ce numéro; il est caractérisé par un col tronconique large à étranglement, et un bord déversé à lèvre triangulaire pendante (fig. $3 n^{\circ}$ 23-25). Malgré la diversité des qualités de pâtes, son origine africaine ne parait pas faire de doute, spécialement dans la région du Sahel. ${ }^{60}$

A Marseille cette amphore ne semble pas apparaître avant le VIe s. (contextes 6, 11 et 13). Elle est le plus souvent associée aux formes $91 \mathrm{C}$ et 99 de sigillée africaine D.

Cette proposition de datation, légèrement plus tardive que celle avancée par Keay (p.394), ${ }^{61}$ va également à l'encontre de celle obtenue sur le site de Carthage où cette amphore est généralement attribuée à l'époque vandale. ${ }^{62}$ En revanche, l'épave de la Palud à Port-Cros, qui livre un chargement important de ce type d'amphore avec différentes variantes de bords, paraît devoir être datée du milieu du VIe s. (sigillées africaines Hayes 88 et 104A). ${ }^{63}$ De même, on retrouve à Roses des associations comparables à celles de Marseille: amphores Keay LXII A et sigillées africaines formes Hayes $91 \mathrm{C}$ et 99.64

Les fouilles de Marseille ont livré un certain nombre de fragments de bords et de fonds avec des traces de poix; les exemplaires complets de l'épave de Filicudi Porto sont également poissés. ${ }^{65}$

Type apparenté au type LXII

Une série assez abondante de bords à section quadrangulaire paraît devoir être rattachée au type Keay LXII (variante E ?) bien que l'étranglement caractéristique du col ne soit plus rappelé ici que par un simple ressaut situé immédiatement sous la lèvre (fig. $3 \mathbf{n}^{\circ}$ 26-29). Certains exemplaires portent un décor peigné sur le col. La pâte est généralement de couleur beige, assez tendre (parfois pulvérulente), avec de grosses inclusions de chaux et de quartz.

Ce type apparaît dans les couches de la fin du VIe ou du VIIe s. (contextes 2 et 8). Il est fréquent sur le site de Sant Antonino di Perti66 en association, comme à Marseille, avec des sigillées africaines formes Hayes 91D, 99B ou C, 105 et 109. Tout se passe comme si l'évolution du type Keay LXII conduisait à une atrophie de la plage située entre le bord et l'étranglement du col.

Les exemplaires de Marseille ne fournisssent aucune information sur le contenu de ces amphores.

Type Keay LX

Nous avons rattaché au type LX de Keay (bien que la pâte de nos exemplaires, rose pâle à surface blanche, ne semble pas correspondre à celle décrite par cet auteur), des amphores caractérisées par un col tronconique sans étranglement et une lèvre triangulaire très effilée (fig. 4 $\left.\mathrm{n}^{\circ} 30\right) \cdot{ }^{67}$

60 D. P. S. Peacock et al., in Amphores romaines et histoire économique (CollEFR 1989) $180 \mathrm{ss.,}$ fig. $6 \mathrm{n}^{\circ} 1$, fig. $7 \mathrm{n}^{\circ} 1-3$, fig. $9 \mathrm{n}^{\circ} 1-4$, fig. $10 \mathrm{n}^{\circ} 1-3$.

61 Nous ne croyons pas non plus à la présence de ce type d'amphore dans le second quart du Ve s. comme cela est suggéré par l'étude du dépotoir de Vila-Roma à Tarragone (Remolà i Vallverdù [supra n.21] 267).

62 Fulford et Peacock (supra n.31) 133 et fig. $40 n^{\circ}$ 68-70; Freed (supra n.8) $86 n^{\circ} 38$.

63 Long et Volpe (supra n.50) 228.

64 J. Nieto Prieto, El edificio " $A$ " de la ciudadela de Roses (La Terra Sigillata Africana) (Centre d'Investigacions Arqueologiques de Girona, Sèrie Monogràfica Nùm. 14, Gerone 1993) 99 sq.

65 C. Albore Livadie, Archeologia subacquea 2 (Boll.d'Arte Suppl. al n²2, 1984) 96. Mais pas ceux, il est vrai fragmentaires, de l'épave de la Palud à Port-Cros.

66 E. Castiglioni et al., ArchMed 19 (1992) 302 ss., pl. VII n 11-14.

67 Notons que ce type n'est illustré que par un seul exemplaire dans la typologie de cet auteur: Keay fig. 131 $n^{\circ} 4$. 


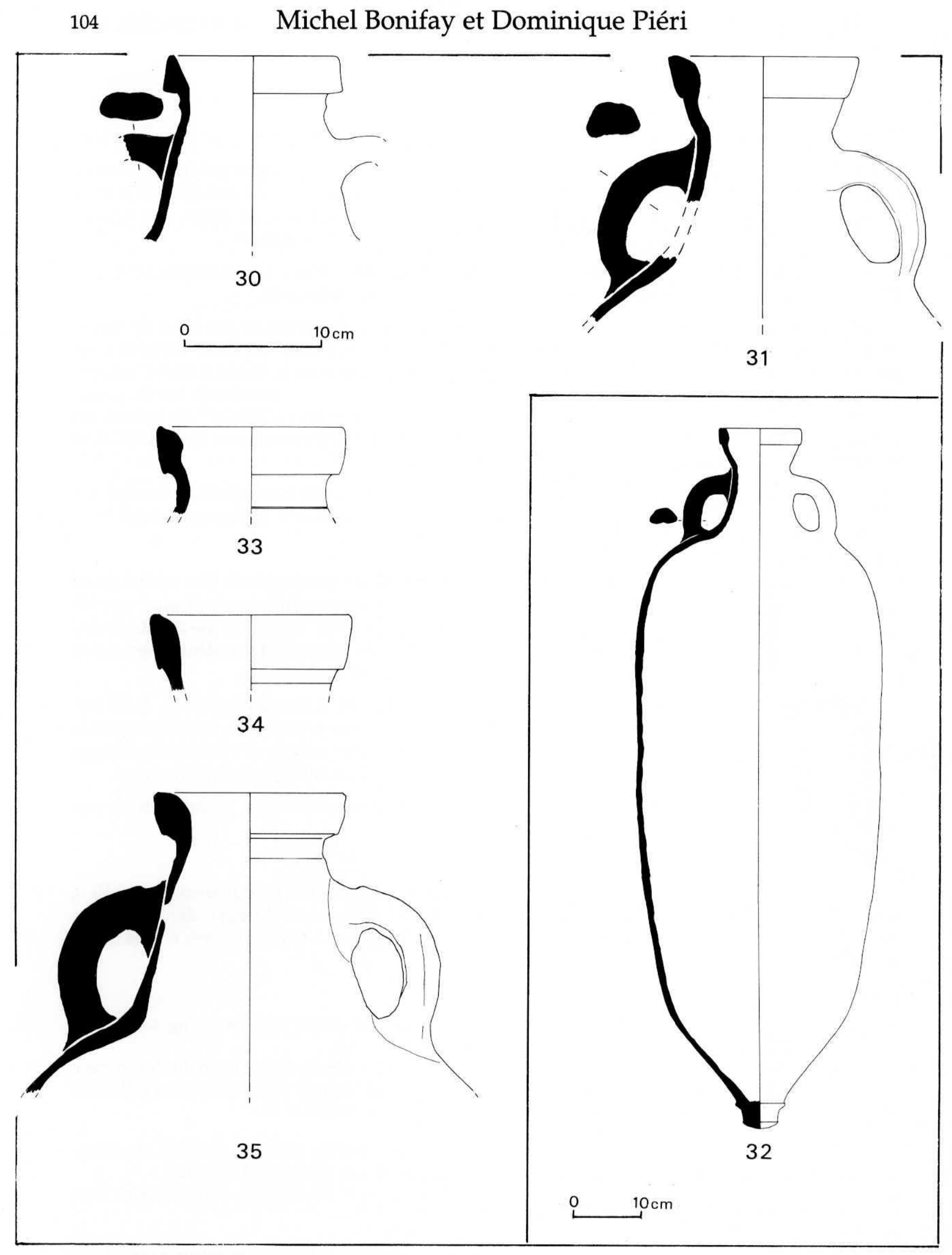

Fig. 4. Amphores africaines. Types Keay LX ( $\left.n^{\circ} 30\right)$, Bonifay 1986 fig. $12 n^{\circ} 55\left(n^{\circ} 31-32\right)$, Keay LXIC $\left(n^{\circ}\right.$ 33-35). 


\section{Typologie et contenu des amphores du Ve au VIIe s. à Marseille 105}

Ces amphores, dont une est reconstituable, ${ }^{68}$ sont présentes dans les niveaux de la fin du VIe et du début du VIIe s. (contexte 2).

Type Bonifay, RAN 1986, fig. 12 n55

Un autre type relativement fréquent à Marseille ne trouve pas de comparaison précise dans la typologie de Keay. Il est caractérisé par un col assez étroit, des anses tombantes de petite taille, et un bord à section convexe dont la lèvre, épaissie, est marquée par un simple ressaut (fig. $4 \mathbf{n}^{\circ}$ 31). La pâte est très particulière, rouge sombre, compacte et dure, avec une surface jaune vif.

Ce type se rencontre à la fin du VIe et au VIIe s. (contextes 2, 3 et 8). Les traces de poix sur l'exemplaire reconstitué (fig. $4 \mathrm{n}^{\circ} 32$ ) semblent exclure un contenu oléagineux bien que cette amphore paraisse avoir été produite dans une région d'Afrique renommée pour son oléiculture. ${ }^{69}$

Type Keay LXI C

Dans la même qualité de pâte que le type précédent, on distingue assez bien un groupe d'amphores à col tronconique sans étranglement et à bord en amande; les anses ont le même profil tombant (fig. $4 n^{\circ}$ 33-35). Le même bord se rencontre sur certains exemplaires classés par Keay dans sa variante LXI C (Keay fig. $133 \mathrm{n}^{\circ} 6$ et 8 ).

Ces amphores sont typiques des contextes de la fin du VIe et du VIIe s. (contextes 2, 8, 16 et 20); aucune indication sur le contenu.



Fig. 5. Amphores africaines. Types Keay LXIA ( $\left.n^{\circ} 36-38\right)$, Keay VIIIA ( $\left.{ }^{\circ} 39\right)$, Keay L $\left(n^{\circ} 40\right)$.

68 Bonifay, RAN 1986, fig. $12 \mathrm{n}^{\circ} 54$.

69 Peacock et al. (supra n.60) fig. $8 n^{\circ} 9$. 


\section{Type Keay LXI A}

L'amphore Keay LXI à bord large en bandeau, dont un exemplaire complet a été découvert naguère en rade de Marseille, ${ }^{70}$ est peu abondante dans les contextes étudiés (fig. $5 \mathbf{n}^{\circ}$ 36-38). $L^{\prime}$ exemplaire $n^{\circ} 38$ présente en outre une pâte très particulière, blanc-rosé, très granuleuse, avec surface verdâtre pulvérulente, qui n'est pas rare sur ce type d'amphore.

Il apparaît sporadiquement à la fin du VIe ou au début du VIIe s. (contexte 2) mais ne devient fréquent qu'au VIIe s. (contextes 3, 15, 19 et 20). Sur l'habitat de Sant Antonino di Perti, il domine, aux côtés de quelques bords apparentés au type Keay LXII (cf. supra) et de nombreux fragments de spatheia miniatures, dans les couches de la phase T4, en association avec des sigillées africaines, formes Hayes 91D, 105 et $109 .{ }^{71}$ Aucune indication de contenu.

Type Keay VIII A

Très proche morphologiquement du type précédent, cette amphore s'en distingue cependant par son bandeau très large mais très peu proéminent, affectant le plus souvent un profil légèrement sinueux, et séparé du col par un simple ressaut au dessin parfois irrégulier (fig. $5 \mathbf{n}^{\circ}$ 39); un exemplaire presque complet est publié à Pieve del Finale (Italie). ${ }^{72}$ La pâte, orange à surface blanche, reste assez proche du standard des productions africaines.

Ce type est complètement absent des niveaux de la fin du VIe et de la première moitié du VIIe s. (contextes $2,3,7,8$ ). Il apparaît en revanche dans des niveaux plus tardifs (contextes 17 et 20) peut-être attribuables à la deuxième moitié du VIIe s., voire même au début du VIIIe s. On le rencontre également dans les couches les plus tardives (phase T2) de l'habitat de hauteur de Sant Antonino di Perti ${ }^{73}$ et sur l'épave de Saint-Gervais II à Fos-sur-Mer ${ }^{74}$ en association, dans les deux cas, avec des monnaies d'Héraclius et avec un mobilier qui peut appartenir à un VIIle s. très avancé. En effet comme le remarque G. Murialdo, ${ }^{75}$ ce type doit être "classé dans la phase la plus tardive de la production africaine" ${ }^{\prime \prime 6}$. On remarque en outre que les amphores retrouvées au large de Fos sont poissées.

Dans les contextes 18 et 20, ce type est associé à des bords de morphologie similaire mais de plus petite taille et plus évasés (fig. $5 n^{\circ} 40$ ), appartenant peut-être à des amphores de type Keay L, ainsi qu'à des fonds ombiliqués appartenant à des amphores globulaires de forme indéterminée. ${ }^{77}$ On retrouve encore la même association de formes à Sant Antonino di Perti. ${ }^{78}$

\section{LES PRODUCTIONS ORIENTALES}

Nous reprendrons l'ensemble des principaux types définis par J. A. Riley à Carthage $\mathrm{e}^{79}$ en les complétant à partir de données nouvelles.

70 B. Liou, Gallia informations 33 (1975) 572 ss. fig.13.

71 Supra n.66, pl. 1-2 et 4-10.

72 G. Murialdo, RevEtLig 54 (1988) 221 ss., fig. $7 \mathrm{n}^{\circ} 6$.

73 E. Castiglioni et al., ArchMed 19 (1992) pl. III n 5-6.

74 Jezegou (supra n.28).

75 Supra n.73.

76 Sur ce site également il succède aux types Keay LXI-LXII.

77 Non dessinés parce que trop fragmentaires.

78 ArchMed 1992, pl. III n ${ }^{\circ}$ 7, pl. IV. Même association encore en Tunisie sur le site de Sidi Jedidi fouillé par Mme A. Ben Abed (Institut National du Patrimoine) et M. M. Fixot (Laboratoire d'Archéologie Médiévale Méditerranéenne); compte-rendus dans MEFRA 104 (1992), 105 (1993) et 106 (1994): la couche de destruction des annexes situées au nord de la basilique orientale livrent des fragments d'amphores Keay LXI, VIIIA et L associés à une monnaie de Constantin IV émise vers 668-674.

79 J. A. Riley in Excavations at Carthage conducted by the University of Michigan 6 (Ann Arbor 1981) 86124. 
Typologie et contenu des amphores du Ve au VIIe s. à Marseille 107

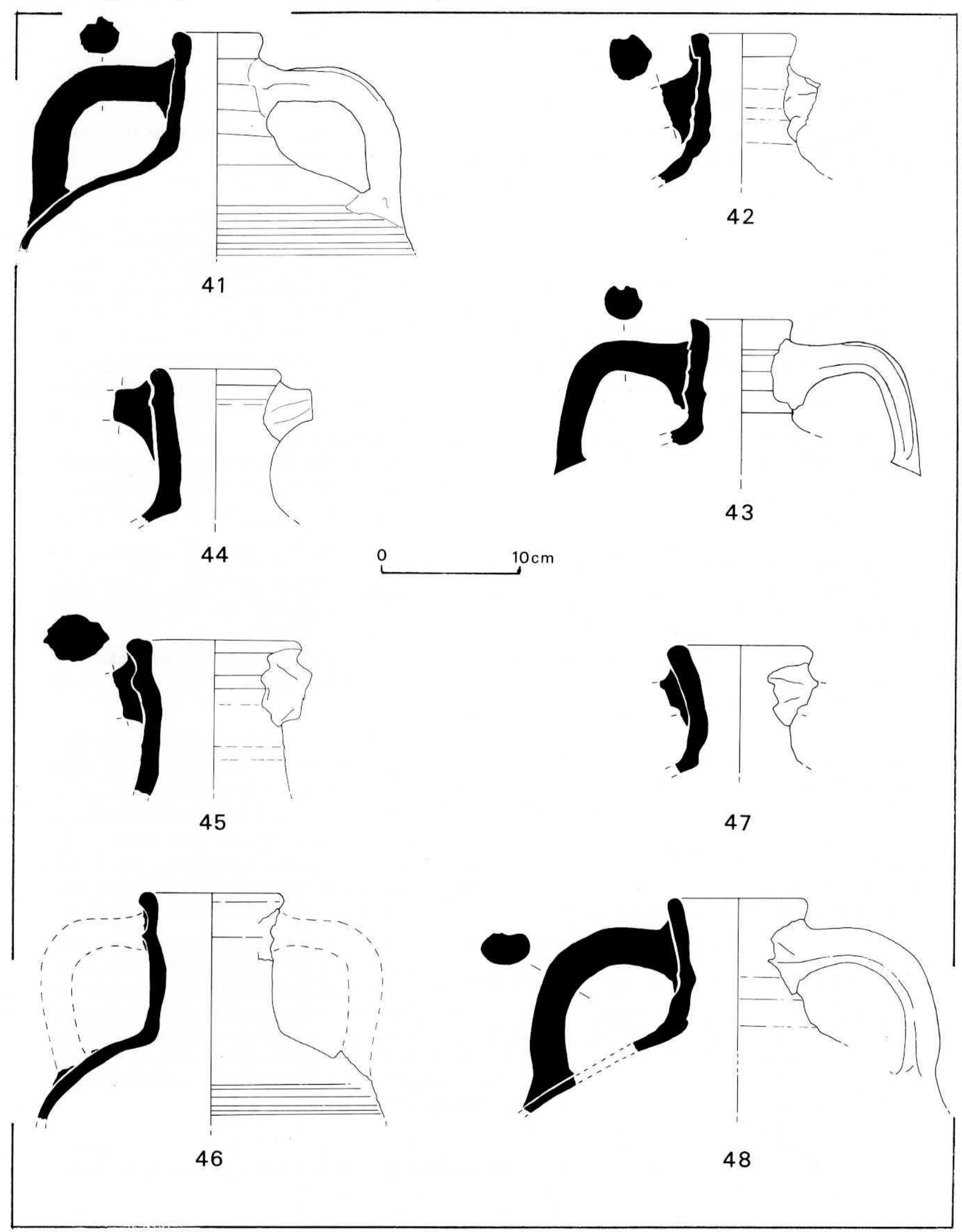

Fig. 6. Amphores orientales. Types L.R.A. 1a ( $\left.\mathrm{n}^{\circ} 41-43\right)$, L.R.A. $1 \mathrm{~b}$ sous-type $1\left(\mathrm{n}^{\circ} 44-46\right)$, L.R.A. 1b sous-type $2\left(n^{\circ} 47-48\right)$. 


\section{Type Late Roman Amphora 1}

En ce qui concerne le premier type de la typologie de Carthage, nous arrivons maintenant à distinguer plusieurs évolutions de la forme:

\section{LRA $1 a$}

Dans les contextes datés des trois premiers quart du Ve s. (contextes 1, 4, 9 et 10), nous trouvons à Marseille un type précoce, que nous appellons LRA 1a et assimilable de façon plus large à la forme Egloff 169 de la typologie égyptienne des Kellia. ${ }^{80}$ Le col est étroit et resserré, plus souvent tronconique que cylindrique, légèrement bosselé. Le bord se compose toujours d'un bandeau dont la hauteur peut varier de 1 à $2 \mathrm{~cm}$, la section de la lèvre pouvant être soit quadrangulaire, soit arrondie; le diamètre externe à l'embouchure varie entre 5 et $7,5 \mathrm{~cm}$. Les anses de section ronde sont coudées et toujours nervurées profondément dans la longueur. Le fond, arrondi, se termine par un bouton. La panse est parcourue par de nombreuses cannelures régulières, en escalier, qui deviennent plus denses sur l'épaulement et à proximité du fond (fig. $6 n^{\circ} 41,42$ et 43 ).

Deux grandes catégories d'argile sont à distinguer pour cette période:

- un premier groupe à pâte sableuse et granuleuse de couleur beige clair à orange comportant de nombreuses inclusions rouges, noires à auréole blanche, de calcaire, de quartz et parfois de mica. La surface est généralement tendre, voire même pulvérulente (la coloration varie du blanc à l'orange vif).

- au second groupe correspond une catégorie d'argile plus fine et plus compacte, constituée de grains de dégraissant en petite quantité. La couleur de la pâte est souvent rose-violet. La surface, plus dure et douce au toucher, a fait l'objet de soins particuliers puisque les canneleures de la panse sont parfaitement régulières et bien dessinées, sans bavures d'argile.

A Marseille, durant le Ve s., l'amphore LRA 1a est le type le plus couramment rencontré; ses proportions variant entre 15 et $28 \%^{81}$ de la totalité des amphores (contextes 1 et 4 ).

\section{LRA $1 b$}

Les niveaux du VIe et du VIIe s. sont marqués par l'apparition apparemment contemporaine de deux nouvelles variantes qui remplacent complètement le type LRA 1a (contextes 2, 7, 8, 19 et 20). Tout d'abord, la forme LRA 1b/sous-type 1 (type Egloff 164 , cf. Egloff pl. $57 \mathrm{n}^{\circ} 4$ ) qui se caractérise par une large embouchure (entre 10 et $12,5 \mathrm{~cm}$ ), une lèvre épaisse en bourrelet (une arête à mi-col rappelle le bandeau du type précédent), une panse trapue de capacité plus modeste (10 litres environ contre 20-25 litres pour le type LRA 1a) (fig. $6 \mathbf{n}^{\circ}$ 44-46). La majorité des exemplaires de ce type retrouvés à Marseille possède une argile très sableuse et pulvérulente de couleur beige-clair ou orangé avec de nombreuses inclusions parfois de grande dimension (contexte 2).

La seconde variante, LRA $1 \mathrm{~b}$ /sous-type 2 , à lèvre déversée vers l'extérieur, se compose d'une argile fine et compacte, lourde et très dure, de couleur beige-clair. La surface est très dure, lisse au toucher, exclusivement de couleur blanche ou beige. Parfois l'argile contient de gros nodules de calcite éclatés en réaction à la cuisson. Les anses sont plus ovales et ne possèdent pratiquement plus de nervure centrale $\left(n^{\circ}\right.$ 47-48). Cette seconde variante est bien attestée à Marseille à la fin $\mathrm{du}$ VIe et au début du VIIe s. (contexte 7) où elle est même majoritaire. Aux Kellia, ce type est daté de la même manière. ${ }^{82}$ Les restes de tituli picti retrouvés à Marseille sur cette deuxième variante changent de couleur: on les trouve peints uniquement en noir.

LRA 1 similis

Dans les contextes du dernier quart du Ve et jusque dans les premières années du VIe s. (contex-

80 M. Egloff, Kellia. La poterie copte (Genève 1977) pl. $58 \mathrm{n}^{\circ} 2$.

81 Bonifay, RAN 1986, 302-3; D. Pieri in Etudes massaliètes 5. 


\section{Typologie et contenu des amphores du Ve au VIIe s. à Marseille 109}



Fig. 7. Amphores orientales. Type L.R.A. 1 similis $\left(\mathrm{n}^{\circ} 49-51\right)$.

tes 9,10 et 12), nous voyons apparaître, à coté de ces types communs, un groupe d'amphores atypiques, illustré par des formes singulières que nous regroupons sous l'appellation LRA 1 similis. Nous pourrions donc situer ici une période de transition entre la fin de la production (ou du moins de l'importation) de la forme LRA 1a et l'évolution finale de la lignée caractérisée par la forme LRA $1 \mathrm{~b}$. Ces variantes particulières du type LRA 1 sont caractérisées pour certains exemplaires par l'augmentation du diamètre du bord qui se situe entre 8 et $10 \mathrm{~cm}$, pour d'autres par la suppression du bandeau soulignant la lèvre (fig. $7 \mathrm{n}^{\circ} 50$ et 51). On note également l'apparition de cols élancés dont certains pourraient se rapprocher de la forme Egloff $168\left(n^{\circ} 49\right)$.

Si l'on connaît bien maintenant la plupart des zones de production de l'amphore LRA 1 , Rhodes, Chypre, côte méridionale de la Turquie et golfe d'Iskenderun ${ }^{83}$ nous ne pouvons encore dire à quel atelier précis appartient telle ou telle catégorie d'argile, en l'absence d'un programme d'analyses pétrologiques systématiques. De même, il est encore difficile d'affirmer la nature des produits transportés dans les amphores LRA 1, le déchiffrement des tituli picti ne nous renseignant en rien sur le lieu précis de la fabrication du vase-conteneur, ni sur son contenu. Cependant, les amphores LRA 1a retrouvées en milieu humide comme dans le port antique de Marseille donnent quelques informations sur les éventuelles denrées transportées puisqu'il apparaît qu'au Ve s. la majorité de ces amphores est poissée; ceci semble donc exclure l'huile des produits les plus fréquemment importés. Au VIe s., nos renseignements sont moins précis. La forme LRA 1 b sous-type 2 , a, en général, conservé son enduit de poix; cela est moins évident pour la forme à pâte sableuse (sous-type 1$) \cdot{ }^{84}$

\section{Type Late Roman Amphora 2}

L'amphore égéenne Carthage LRA 2 n'apparaît à Marseille qu'à partir des premières années

82 F. Bonnet in R. Kasser, Survey archéologique des Kellia (Basse-Egypte), rapport de la campagne 1981 (Louvain 1983) $440 n^{\circ} 71$.

83 J.-Y. Empereur et M. Picon in Amphores romaines et histoire économique 236-43.

84 Cependant les exemplaires provenant de l'épave de la Palud sont poissés (Long et Volpe [supra n.50] 223). Nous avons pu également observer, sur les sites de Fos-sur-Mer et de Port-Vendres, plusieurs amphores du même type poissées (matériel en cours d'étude). 


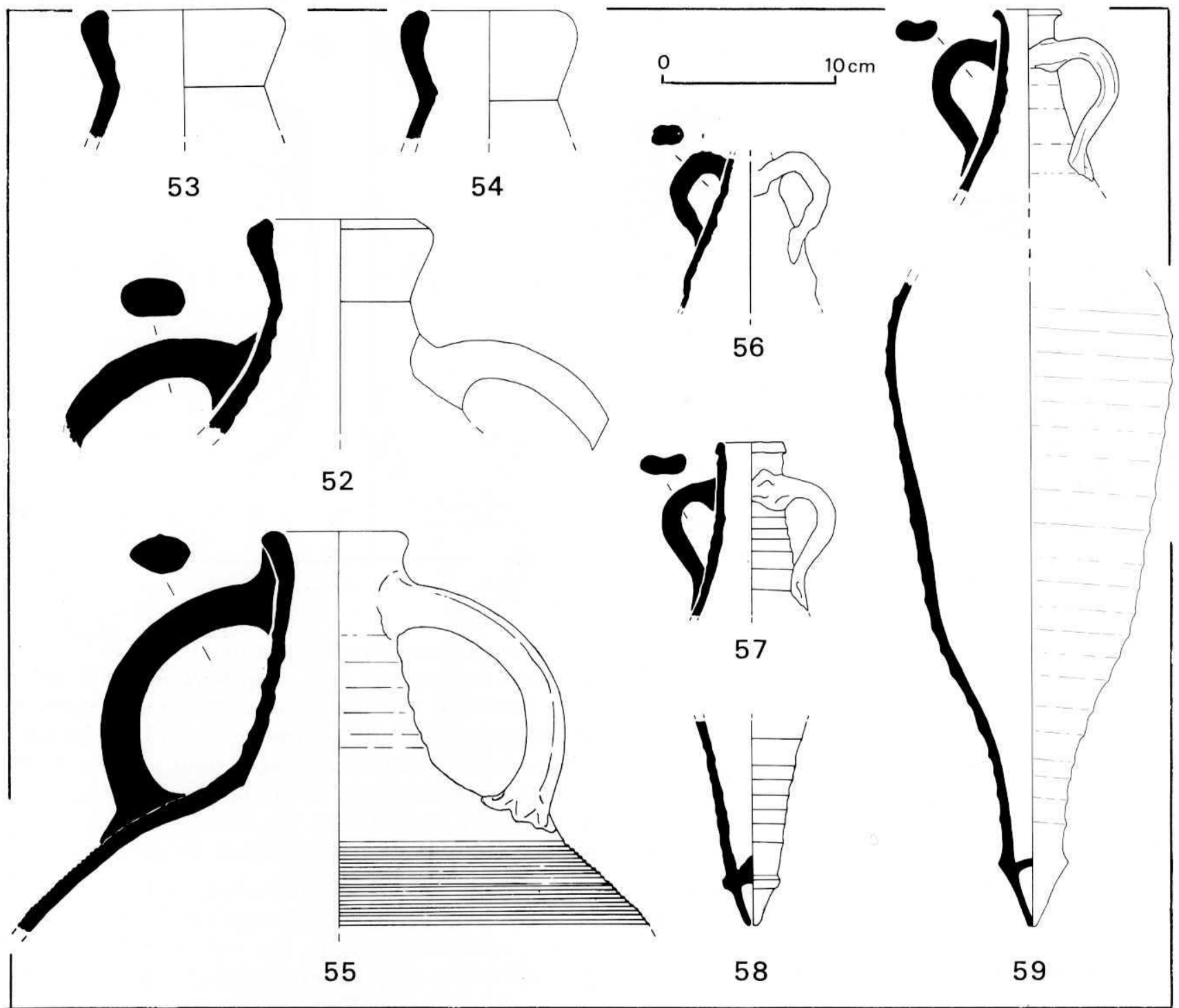

Fig. 8. Amphores orientales. Types L.R.A. 2 sous-type 1 ( $n^{\circ}$ 52-54), L.R.A. 2 sous-type 2 ( ${ }^{\circ}$ 55), L.R.A. 3 variante $1\left(n^{\circ} 56\right)$, L.R.A. 3 variante $2\left(n^{\circ} 57-59\right)$.

du VIe s. sous deux variantes, dissociées essentiellement à partir de la morphologie du col et du bord. La première, LRA 2/sous-type 1, possède un col tronconique surmonté d'un bord en entonnoir (fig. $\left.8 \mathbf{n}^{\circ} \mathbf{5 2}, \mathbf{5 3}, \mathbf{5 4}\right)$. Cette forme, la plus courante, n'existe que dans des proportions toujours modestes (contextes $2,6,7,8,14$ et 15). La seconde (sous-type 2), plus tardive (VIIe s.?), est formée d'un bord vertical terminé par une petite lèvre courte $\left(\mathbf{n}^{\circ}{ }^{55}\right){ }^{85}$ Le décor, ainsi que son développement sur la panse, peut varier: le plus souvent profondément strié et ondulé sur la première variante, il peut également contenir de fines cannelures plates ou de simples stries faites au peigne sur la deuxième variante.

Ce col est hors contexte mais un exemplaire comparable provient de la fouille de la nécropole tardive du fort Ganteaume menée en 1988 par l'Equipe Municipale d'Archéologie. Ce col a été retrouvé dans une tombe datée du VIIe s. (?), où il servait vraisemblablement de conduit à libation (L.-F. Gantès et M. Moliner (Dir.), Marseille, Itinéraire d'une mémoire, Cinq années d'archéologie municipale [Marseille 1990] 63-64). 


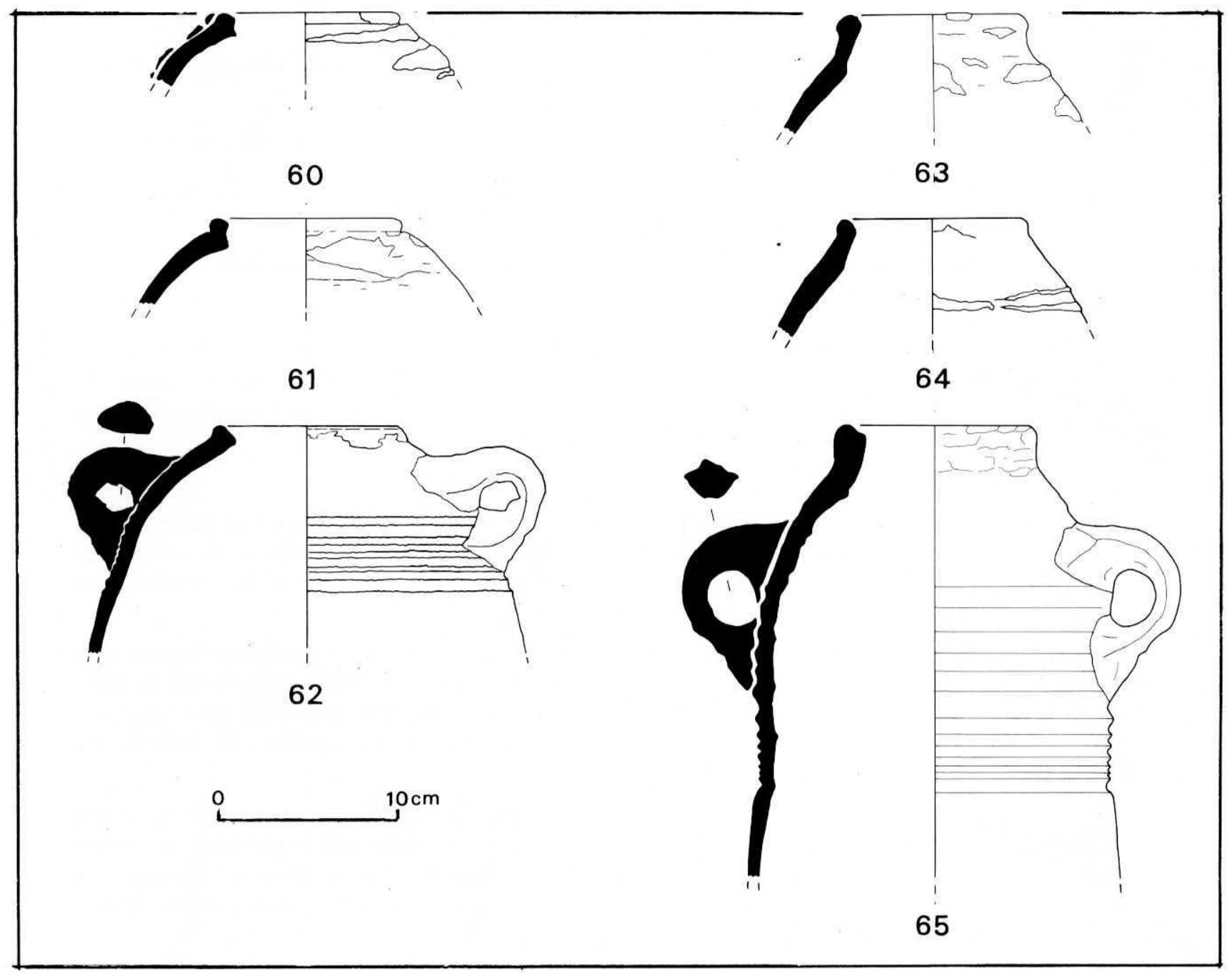

Fig. 9. Amphores orientales. Types L.R.A. $4 a\left(n^{\circ} 60-62\right)$, L.R.A. $4 b\left(n^{\circ} 63-65\right)$.

Contenu: incertain; quelques fragments ont conservé des restes de résine.

\section{Type Late Roman Amphora 3}

Nous arrivons à bien distinguer la présence de deux variantes du type LRA 3 de Riley, qui se succèdent dans le temps. La première variante, qui se rapproche du type Robinson M 307 de l'Agora d'Athènes, ${ }^{86}$ se distingue par un col court, des épaules carénées, de petites anses fines ainsi qu'un fond ombiliqué (fig. $8 \mathrm{n}^{\circ}$ 56). Elle apparaît à Marseille dans le courant du Ve s., mais toujours en infime quantité (contexte 1). Ailleurs, comme à Arles, on la rencontre dès le début du Ve s. ${ }^{87}$

La seconde, qui ne semble pas apparaître à Marseille avant le second quart du Ve s. (contextes $1,4,5$ et 12), s'apparente à la forme Robinson M 373. Le col est plus long, les épaules adoucies, les anses larges, un fond creux et pointu ( $n^{\circ} \mathbf{5 7}, 58$ et 59$)$. Plus rare dans les contextes du second quartmilieu du VIe s. et absente à la fin de ce siècle, sa commercialisation à Marseille cesse

86 H. S. Robinson, The Athenian Agora V. Pottery of the Roman period (Princeton 1959).

87 Congès et Leguilloux (supra n.17). 
vraisemblablement vers cette date. Son origine demeure toujours imprécise même si le triangle Halicarnasse-Bodrum-Sardes paraît des plus probables. ${ }^{88}$

A Marseille, les exemplaires trouvés dans le port antique (contextes 1 et 4 ) sont systématiquement poissés (contenu: vin ou garum?).

\section{Type Late Roman Amphora 4 (amphore dite de Gaza)}

L'amphore oblongue dite 'de Gaza' connaît également une évolution remarquable.

\section{LRA 4 a (Zemer $53^{89}$ )}

Elle est d'abord globulaire dans les contextes datés du Ve s. (contextes 1, 4, 12). Le bord, marqué par une lèvre en bourrelet mince à ressaut interne, se détache à peine du corps de l'amphore (il n'y a pas de col); les anses soudées sur des épaules arrondies sont proches du bord (fig. $9 \mathrm{n}^{\circ}$ 60-62).

\section{LRA 4 b (Zemer 51)}

Cette amphore augmente de capacité au VIe s. (contextes 2, 6, 7, 8, 14): son corps s'allonge, les épaules deviennent tombantes, la lèvre, en devenant plus haute, forme une amorce de col ( $\mathbf{n}^{\circ}$ 6365). A la fin du VIe s., la variante b est la forme la mieux représentée parmi les productions orientales.

Très fréquente dans les niveaux tardifs marseillais, l'amphore de Gaza a également connu une très large diffusion en Gaule du Ve jusque dans le courant du VIIe s. ${ }^{90} \mathrm{Il}$ faut aujourd'hui revenir, depuis les récentes recherches de J.-Y. Empereur, sur la thèse d'une fabrication de cette amphore dans le delta du Nil, son aire de production s'étendant uniquement de la partie orientale du Nil jusqu'à Gaza. ${ }^{91}$

Alors même qu'existent des textes sur l'exportation du vin de Gaza au VIe s., il est remarquable que les amphores LRA 4 trouvées à Marseille ne sont pas toutes poissées; le célèbre vin de Gaza semble ne pas avoir été la seule seule denrée exportée dans ce récipient. D'après les analyses physico-chimiques, c'est la seule amphore orientale tardive susceptible d'avoir contenu un liquide oléagineux. ${ }^{92}$

\section{Type Late Roman Amphora 5}

La forme LRA 5 n'apparait pas à Marseille avant le dernier tiers du Ve s. (contextes 12 et 13); d'abord dans une variante particulière - un petit module à bord très court (fig. $10 n^{\circ} 66$ et 67 ); puis vers la fin du VIe et au VIIe s. dans la variante commune de l'amphore à pâte sableuse orangée ou blanchâtre (contextes 2, 7, 8, 15). Quelques fragments seulement semblent provenir $\mathrm{d}^{\prime}$ Egypte et notamment d'Abou Mina, ${ }^{93}$ la majorité appartenant à l'aire syro-palestinienne $\left(\mathbf{n}^{\circ}\right.$ 68-71). A Marseille-Bourse, vers la fin du VIe s., c'est, avec la forme LRA 4b, le type oriental le mieux attesté: elle représente à elle seule près de $12 \%{ }^{94}$ de la totalité des fragments d'amphores (contextes 2, 7,8).

88 C. Panella, in Recherches sur les amphores grecques (BCH Suppl. 13, 1986) 614-15.

89 A. Zemer, Storage jars in ancient sea trade (Haifa 1977).

90 Bonifay et Villedieu (supra n.6) 27-29.

91 J.-Y. Empereur et M. Picon in Cahiers de la céramique égyptienne 3 (1992) 145-52.

92 Nous ne disposons pour cette amphore d'aucune information nouvelle sur un éventuel contenu oléagineux depuis les analyses effectuées par Rothschild-Boros qui avait mis en évidence, à partir d'échantillons provenant des remblais de la Schola Praeconum à Rome, la présence d'huile de sésame (M. C. RothschildBoros in Archaeology and Italian society II [BAR S 102, Oxford 1981] 83).

93 Nous remercions chaleureusement $P$. Ballet d'avoir bien voulu nous confirmer ces hypothèses.

94 Bonifay, RAN 1986, 302-3; Piéri in Etudes massaliètes 5. 


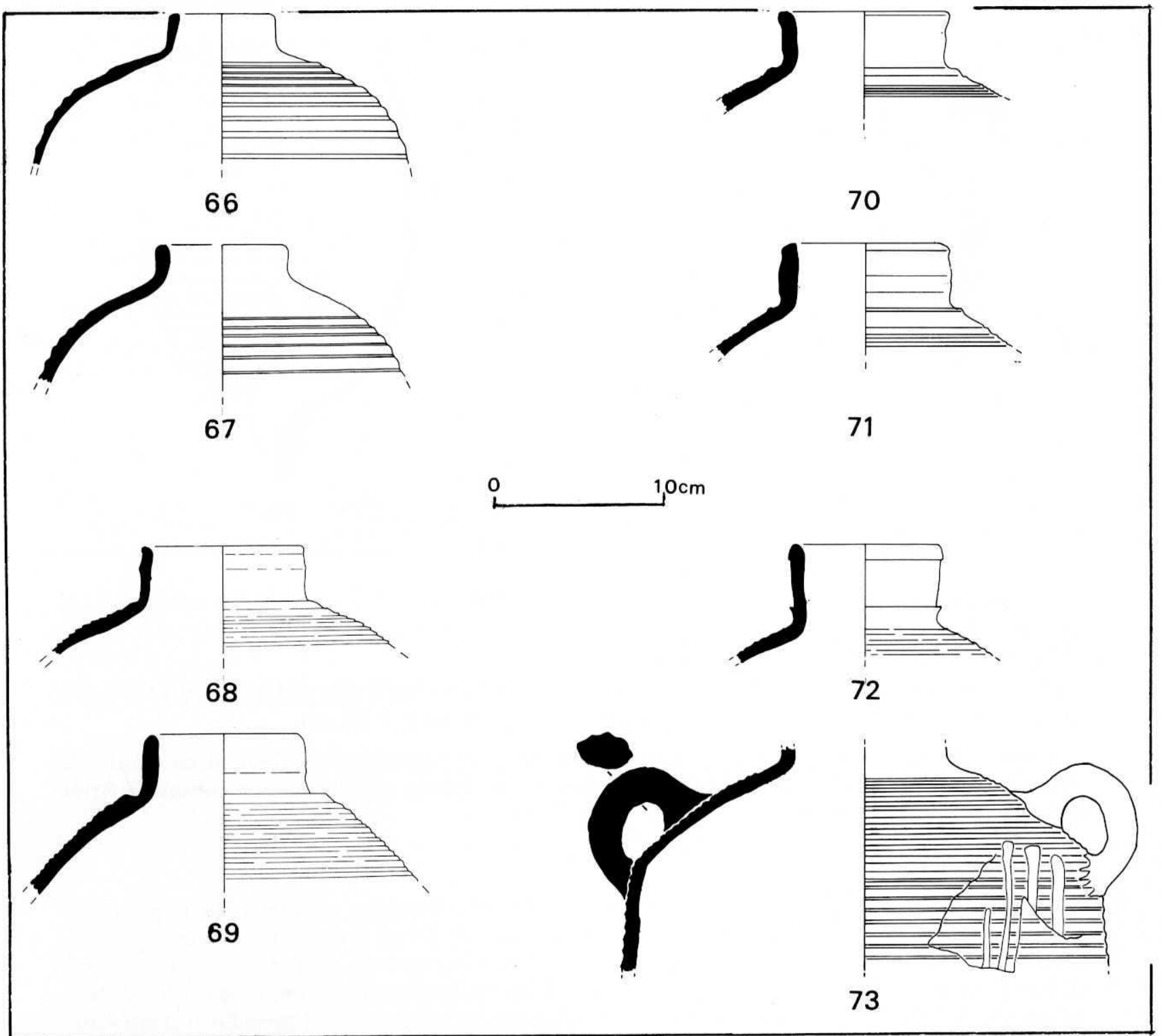

Fig. 10. Amphores orientales. Types L.R.A. 5 variante $1\left(n^{\circ} 66-67\right)$, L.R.A. 5 variante $2\left(n^{\circ} 68-71\right)$, L.R.A. $6\left(\mathrm{n}^{\circ} 72-73\right)$.

La poix, retrouvée sur plusieurs exemplaires des contextes 7 et 8 , laisse entendre que l'amphore 'en forme de sac' (bag-shaped) contenait un de ces fameux vins palestiniens tant appréciés aux VIe et VIIe $\mathrm{s} .{ }^{95}$

\section{Type Late Roman Amphora 6}

La forme LRA 6 est très rare à Marseille et plus généralement en Gaule (fig. $10 \mathbf{n}^{\circ} 72$ et 73 ). Elle se distingue du type LRA 5 par la texture de son argile qui est plus dure et plus compacte (grise en surface et rouge à brune dans le coeur) ainsi que par sa morphologie (bord haut, épaules cassées); c'est une amphore d'assez grande contenance. Ce type n'est attesté à Marseille que dans des niveaux datés du Ve s. (contextes 1,4 et 10 ).

95 J.-P. Sodini in Mémoires d'Euphrate et d'Arabie (ouvrage collectif) (Paris 1991) p 152. 


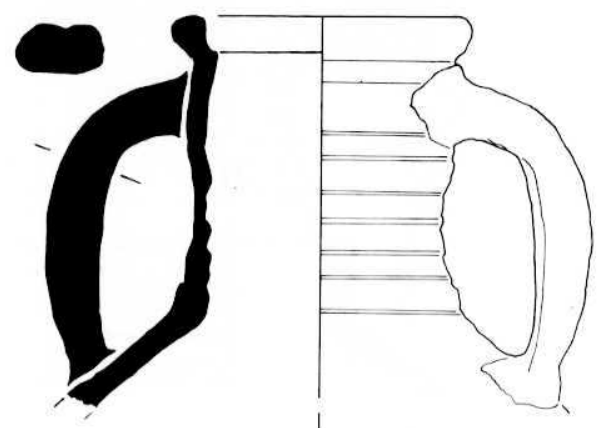

74


Fig. 11. Amphores orientales. Type Robinson M 273. ( $\left.\mathrm{n}^{\circ} 74-75\right)$

\section{Type Late Roman Amphora 7}

A Marseille, on ne trouve que la variante la plus tardive, c'est à dire la forme Egloff 172-174 caractéristique de la fin du VIe et du début du VIle s. (contextes 2, 7, 19, 20).

Dans l'Antiquité tardive, les amphores égyptiennes apparaissent timidement en Gaule au début du Ve s. et uniquement sur les sites proches du littoral méditerranéen comme à Arles (Congès 1991, fig. $\left.8 \mathrm{n}^{\circ} 33\right)^{96}$.

\section{Type Robinson M 273}

L'amphore M 273 de l'Agora d'Athènes est probablement d'origine orientale. Elle regroupe de nombreuses variantes hétérogènes qui n'ont pas encore fait l'objet d'étude typologique précise. $C^{\prime}$ est une amphore à col cylindrique large, occasionnellement cannelé, avec des anses massives à section ovale et une panse ovoïde cannelée; un petit fond pointu termine l'amphore (fig. $11 \mathbf{n}^{\circ} 74$ et 75). Sa pâte est rouge ou orange très micacée. Moyennement représentée à Marseille, il semble que ce conteneur ait surtout intéressé le Ve s. (contextes 1,4,12). L'intérieur des vases est très souvent poissé. Contenu: incertain (vin ?).

\section{LES PRODUCTIONS ITALIQUES}

La seule forme italique tardive attestée à ce jour à Marseille est l'amphore Keay LII (ou Robinson M 234). Relativement abondante, elle peut parfois atteindre $14 \%$ du total des amphores dans certains contextes. ${ }^{97}$

Après de longues controverses à propos de l'origine de la production, on considère maintenant le type comme originaire de Calabre,; ${ }^{98}$ notons cependant que les cols 78 et 79 portent des inscrip-

96 Congès (supra n.17) fig. $8 \mathrm{n}^{\circ}$ 33. Il s'agit d'abord du type Egloff 177 au début du Ve s. (Arles), puis de la forme 172-74 présente à Marseille à partir de la fin du VIe $s$.

97 Bonifay, RAN 1986, 302-3; Piéri in Etudes massaliètes 5.

98 P. Arthur, JRA 2 (1989) 133-42. 


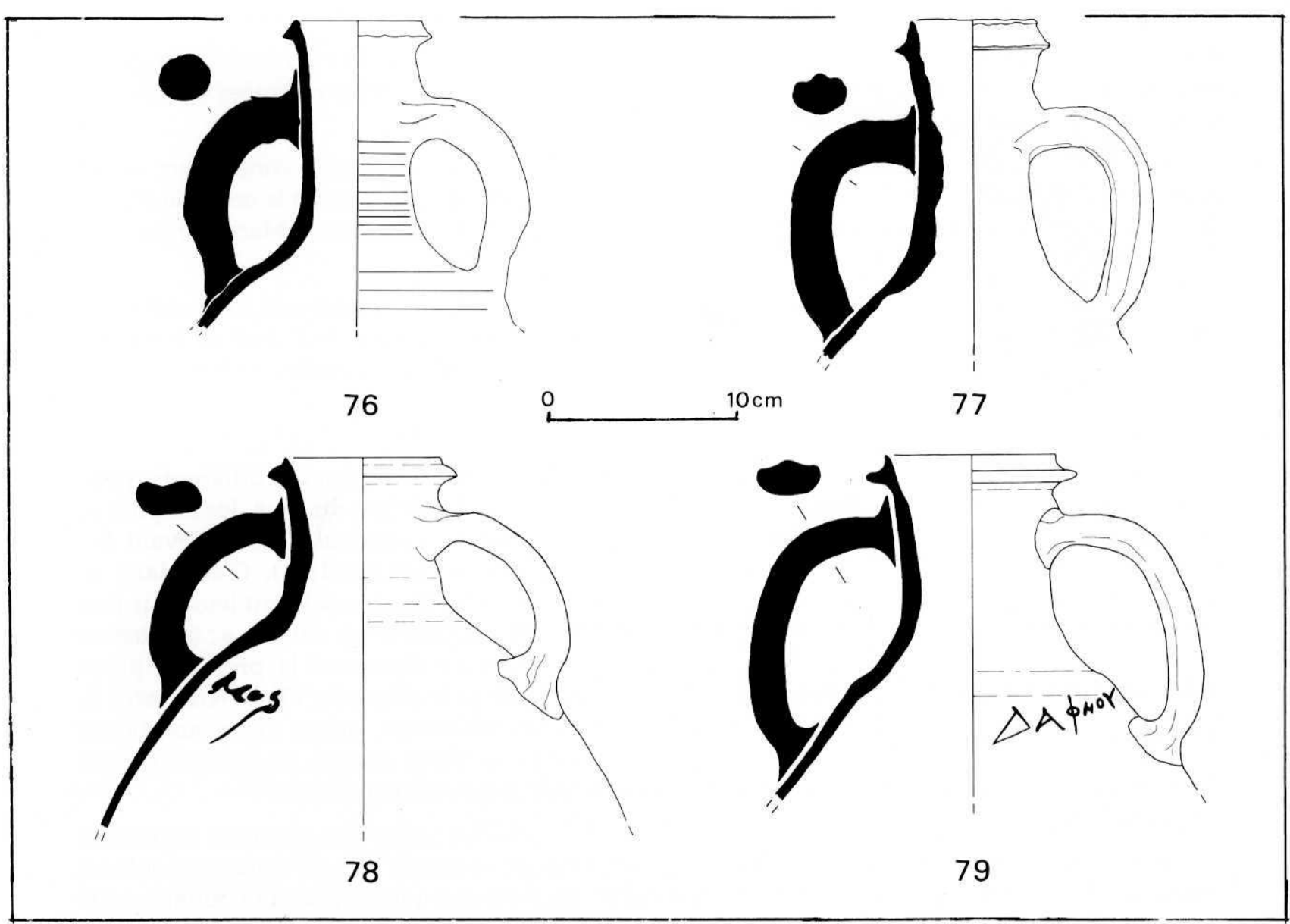

Fig.12. Amphores italiques. Type Keay LII sous-type 1 ( $\left.n^{\circ} 76-77\right)$, sous-type 2 ( $\left.{ }^{\circ} 78\right)$, sous-type 3 ( $\left.n^{\circ} 79\right)$.

tions en grec. Les exemplaires marseillais présentent une assez grande variété de pâtes qui ont toutefois en commun une forte proportion de mica (bien visible en surface), un aspect assez grossier et une teinte dominante beige. Il ne semble pas y avoir de correspondance entre ces variations de pâtes et les variations observées dans la typologie.

En effet, nous croyons pouvoir distinguer à Marseille deux ou trois sous-types principaux: - le sous-type 1 (fig.12 $\mathbf{n}^{\circ}$ 76-77) se caractérise par un long col cylindrique, légèrement tronconique et étroit; une lèvre triangulaire marquée par une gorge horizontale, une panse piriforme légèrement cannelée, ainsi que des anses à section ronde parfois nervurées. Le diamètre à l'ouverture est en moyenne de $5 \mathrm{~cm}$. Un exemplaire complet découvert dans le contexte 1 mesure $48 \mathrm{~cm}$ de haut (dessin publié dans JRA 2 [1989] 140 fig. $1 \mathrm{n}^{\circ} 1$ ). Des exemplaires très proches découverts à Rome sont datés du Ve s. ${ }^{99}$

- le sous-type 2 se distingue par un col plus court et plus large que sur les exemplaires précédents (diam.: $8 \mathrm{~cm}$ env.). La forme est globulaire, les anses rubanées sont garnies d'une nervure centrale assez profonde, la surface de la panse n'est pas cannelée. Quant au bord, il se compose d'une lèvre aplatie à section triangulaire $\left(n^{\circ} \mathbf{7 8}\right)$. Cette variante se rapproche d'un exemplaire de la fin du Ve s. trouvé à Rome ${ }^{100}$ ou encore de l'exemplaire du baptistère d'Albenga. ${ }^{101}$

99 A. Carignani et F. Pacetti, in Recherches sur la céramique byzantine (BCH Suppl. 18, 1989) 5 ss., fig.4.

100 Arthur (supra n.98) 140 et fig. $1 \mathrm{n}^{\circ} 8$.

101 Pallarès (supra n.42) fig. $28 \mathrm{n}^{\circ} 25$. 
- le type 3 se distingue du précédent par son bord, élancé vers l'extérieur et creusé d'une gorge externe très profonde. Deux petites nervures parallèles garnissent les anses rubanées $\left(n^{\circ} 79\right)$. Un exemplaire comparable provient de Naples. ${ }^{102}$

Le type 1 est celui qui est le plus couramment rencontré à Marseille au second quart ou au milieu du Ve s. (contexte 1). Les types 2 et 3 semblent plutôt caractéristiques de la seconde moitié du Ve s. (contexte 4). L'amphore Keay LII ne paraît plus avoir été importée à Marseille au delà du début du VIe s.

Etant donné que tous les exemplaires retrouvés en milieu humide sont poissés, nous estimons que ce conteneur était destiné au transport du vin, ce qui conforte la thèse de P. Arthur destinant cette amphore au transport du vin du Bruttium connu depuis la plus haute Antiquité. ${ }^{103}$

\section{CONCLUSION}

Nous ne reviendrons pas en détail sur les données statistiques concernant ces différents types d'amphores. ${ }^{104}$ Rappelons seulement ${ }^{105}$ que dans les contextes du milieu du Ve s. les amphores orientales occupent la première place (entre 33 et $37 \%$ dans les contextes 1 et 4 ) devant les amphores africaines (22-27\%) et les amphores italiques Keay LII (10-16\%). C'est dans le contexte 12, daté du dernier tiers du Ve s., que les amphores orientales connaissent leur plus fort pourcentage $((47 \%)$ devant les amphores africaines $(27 \%)$ et italiques $(7 \%)$. Au VIe s., (contextes $5,6,13)$ la tendance semble s'inverser, les amphores africaines reprenant la première place (entre 30 et $68 \%$ ) devant les orientales (16 à $25 \%$ ). La fin du VIe et le début du VIIe s. (contextes 2 , $7,8)$, confirment cette tendance avec $40-50 \%$ d'amphores africaines, même si les amphores orientales demeurent encore bien représentées (19-30\%). Pour le VIIe s. avancé, on manque encore de données fiables mais les amphores africaines paraissent toujours majoritaires. ${ }^{106}$

Du point de vue typo-chronologique, on croit déceler parmi les amphores africaines importées à Marseille une certaine stagnation du répertoire de formes au cours du Ve s., ${ }^{107}$ qui n'est pas sans rappeler celle des formes de sigillée africaine, à condition, bien sûr, que l'on accepte de repousser au VIe s. l'apparition des formes Hayes 88, 91C, $99 .{ }^{108}$ En effet, dans notre ville, ces formes sont clairement contemporaines du type Keay LXIIA qui marque le début de l'importation d'une nouvelle génération d'amphores cylindriques de grande dimension caractérisées par leur fond à renflement annulaire. Ce problème de chronologie absolue devient encore plus difficile avec les types les plus tardifs de cette production: s'il semble que les types Keay XLI puis VIIIA succèdent au type Keay LXIIA, on hésite encore sur leur datation précise. L'apparition timide du type Keay LXI et l'absence totale du type VIIIA dans les contextes de la fin du VIe $s$. et de la première moitié du VIIe s.(contextes 2, 3, 7,8) militent en faveur d'une date extrêmement tardive. Le type VIIIA serait peut-être à placer au milieu ou dans la deuxième moitié du VIIe siècle, voire même plus tard, car il est associé à des amphores globulaires à fond ombiliqué qui rappellent ou annoncent le type désormais bien connu dans toute la péninsule italique pour les VIIIe et IXe s. ${ }^{109}$

102 Arthur (supra n.98) fig. $1 \mathrm{n}^{\circ} 2$.

103 Arthur (supra n.98) 138.

104 Bonifay, RAN 1986; Boiron et al. in Etudes massaliètes 5; Piéri in Etudes massaliètes 5.

105 Les comptages qui suivent sont exprimés en nombre de bords-fonds-anses, dans un souci d'homogénéité avec ceux présentés par Tomber, JRA 6 (1993).

106 Comme sur le site de Sant Antonino di Perti (ArchMed 19 [1992]).

107 Même observation chez Panella (supra n.13) 182, paragraphe C.

108 Selon la première hypothèse de J. W. Hayes, Late Roman pottery (London 1972). Contra: S. Tortorella in Società romana e impero tardoantico, III, Le merci, gli insediamenti (Rome 1986) p.211-25.

109 L. Paroli in L. Paroli et P. Delogu, La storia economica di Roma nell'alto Medioevo alla luce dei recenti scavi archeologici, Actes du séminaire (Rome, 2-3 avril 1992), Biblioteca di Archeologia Medievale (Florence 1993) 243. Il en est de même dans le sud de la France sur le site du Bouquet, San-Peyre à Suzon (Gard) où l'on trouve des amphores cylindriques Keay LXI et VIIIA associées à des amphores à corps 
Pour les amphores orientales, il semble nécessaire de s'affranchir un peu de la typologie élaborée en 1976 par Riley à Carthage, qui, si elle a permis de mettre en évidence, notamment à Marseille, un matériel jusqu'alors méconnu, ne parvient plus aujourd'hui à traduire la complexité des variations typo-chronologiques. Il devient en effet indispensable de distinguer deux, voire trois générations au sein du type Late Roman Amphora 1, la variante Egloff 169 étant caractéristique du Ve s. et la variante Egloff 164 n'apparaissant pas avant le VIe siècle. Il en est de même pour le type Late Roman Amphora 4 dont, au Ve s., on ne connaît à Marseille que la variante Zeemer 53 et, au VIe s., que la variante Zeemer 51. Enfin les amphores 'en forme de sac' qui apparaissent dans la deuxième moitié du Ve s. (contexte 12) n'ont que peu de rapport, dans le détail, avec le type classique Late Roman Amphora 5 particulièrement abondant à Marseille à la fin du VIe et au début du VIIe s. (contextes 2, 7, 8).

Du point de vue de la nature des contenus, on est frappé par la proportion d'amphores poissées qui ne peuvent donc avoir été utilisées pour la commercialisation de l'huile. On pouvait s'attendre à une telle observation pour les amphores d'origine orientale, encore que le type Late Roman Amphora 1 ait été parfois assigné au transport de l'huile; ${ }^{110}$ en revanche, alors que le vin de Gaza est mentionné par d'assez nombreux textes, y compris en Gaule mérovingienne, ${ }^{111}$ on constate que les exemplaires découverts à Marseille ne sont pas systématiquement poissés. Mais il est bien plus étonnant de rencontrer un grand nombre d'amphores africaines poissées car la large diffusion de ces conteneurs a le plus souvent été considérée comme liée au développement de l'oléiculture, bien attesté par les textes dans cette province de $l^{\prime} E m p i r e .{ }^{112}$ Or une enquête menée récemment sur une plus grande échelle permet de supposer que la majorité des amphores africaines importées en Gaule du Sud du IIe au VIIe s. ne transportait pas d'huile. ${ }^{113}$ Mais on hésite encore sur la nature des contenus: salaisons ou vin?

Les données exposées ici de manière très schématique doivent être considérées comme des hypothèses de travail que nous soumettons à l'examen critique de nos collègues oeuvrant en d'autres points du bassin méditerranéen. Il est par ailleurs probable que la poursuite des recherches à Marseille permettra d'apporter rapidement de nouveaux résultats. ${ }^{114}$

CNRS, Centre Camille-Jullian et Recherches d'Antiquités Africaines (UMR 9968), Université de Provence (M.B.); 37 Cours Lieutaud, Marseille (D.P.).

\footnotetext{
globulaire dans un ensemble clos (incendie) daté, par une inscription arabe sur tablette, de la deuxième moitié du VIIe ou de la première moitié du VIIIe siècle (C. Pellecuer et J.-M. Pène, Archéologie du Midi Médiéval 11 [1993] p. 151). La présence dans ce contexte d'une lampe africaine de type Atlante X ne doit pas étonner (C. Panella in Merci e scambi nel Mediterraneo tardoantico (Storia di Roma III, 2 ) [Turin 1993] 674).

110 Riley (supra n.26) 215.

111 Cf. les références rassemblées par Riley (supra n.26) 222.

112 A. Carandini in StMisc 15 (1970) 106.

113 Ben Lazreg et al. (supra n.7).

114 Nous remercions M. G. Murialdo de nous avoir communiqué son manuscrit "Anfore tardoantiche nel Finale (VI-VII secolo)", à paraître dans la Revue d'Etudes Ligures, Mélanges offerts à Paul-Albert Février. Menée d'une manière tout à fait indépendante à partir d'un matériel différent, cette étude confirme néanmoins, dans ses grande lignes, la chronologie relative des différents types d'amphores telle qu'elle est apparue à Marseille et surtout la complète pour les périodes les plus récentes.
} 


\section{ANNEXE 1}

\section{PROVENANCE DES OBJETS ILLUSTRÉS.}

\section{Liste des chantiers:}

- La Bourse: fouilles 1979-1984. Cf. Bonifay, RAN 1983.

- Rue du Bon-Jésus: fouilles 1992. Responsable: Patrick Reynaud (AFAN).

- Cap Titol: fouilles 1993. Responsable: Roger Boiron (AFÂN).

- Rue de la Cathédrale: fouilles 1986. Responsables: Lucien-François Gantès et Manuel Moliner (Equipe Municipale d'Archéologie). Cf. Gantès-Moliner (supra n.85) 11-18.

- Place Jules Verne: fouilles 1992-1993. Responsable: Antoinette Hesnard (CNRS-UMR 9968). Cf. Hesnard, CRAI 1994.

$\mathrm{N}^{\circ} 1$ à 3: Marseille. La Bourse. Contexte 1.

$\mathrm{N}^{\circ}$ 4: Marseille. Rue du Bon-Jésus. Contexte 12.

$N^{\circ}$ 5: Marseille. La Bourse. Hors contexte.

$\mathrm{N}^{\circ}$ 6: Marseille. Place Jules Verne. Contexte 18

$N^{\circ} 7$ à 11: Marseille. La Bourse. Contexte 1.

$\mathrm{N}^{\circ} 12$ : Site de Yonga (Tunisie). Programme tunisofrançais d'étude du littoral tunisien (prospection).

$\mathrm{N}^{\circ} 13$ à 15: Marseille. Rue du Bon-Jésus. Contexte 12.

$N^{\circ}$ 16: Marseille. Cap Titol. Contexte 13.

$N^{\circ}$ 17: Marseille. Rue du Bon-Jésus. Contexte 12.

$N^{\circ} 18$ à 20: Marseille. La Bourse. Contexte 6.

$\mathrm{N}^{\circ} 21$ à 23: Marseille. Cap Titol. Contexte 13.

$\mathrm{N}^{\circ} 24$ et 25: Marseille. La Bourse. Contexte 6.

$\mathrm{N}^{\circ}$ 26: Marseille. La Bourse. Contexte 2.

$\mathrm{N}^{\circ} 27$-28: Marseille. La Bourse. Contexte 8.

$N^{\circ}$ 29: Marseille. La Bourse. Contexte 7.

$\mathrm{N}^{\circ}$ 30: Marseille. La Bourse. Contexte 2.

$N^{\circ}$ 31: Marseille. La Bourse. Contexte 3.

$N^{\circ}$ 32: Marseille. La Bourse. Contexte 2.
$\mathrm{N}^{\circ} 33$ et 34: Marseille. La Bourse. Contexte 8. $\mathrm{N}^{\circ}$ 35-36: Marseille. La Bourse. Contexte 2.

$\mathrm{N}^{\circ}$ 37:Marseille. Place Jules Verne. Contexte 17.

$\mathrm{N}^{\circ}$ 38: Marseille. La Bourse. Contexte 3.

$\mathrm{N}^{\circ}$ 39-40: Marseille. Place Jules Verne. Contexte 17.

$\mathrm{N}^{\circ}$ 41-42: Marseille. La Bourse. Contexte 1.

$\mathrm{N}^{\circ}$ 43: Marseille. La Bourse. Contexte 4.

$\mathrm{N}^{\circ}$ 44: Marseille. La Bourse. Contexte 5.

$\mathrm{N}^{\circ}$ 45: Marseille. Place Jules Verne. Contexte 20.

$\mathrm{N}^{\circ}$ 46: Marseille. La Bourse. Contexte 2.

$N^{\circ}$ 47: Marseille. La Bourse. Contexte 8.

$N^{\circ}$ 48: Marseille. La Bourse. Contexte 8.

$\mathrm{N}^{\circ}$ 49: Marseille. Rue du Bon-Jésus. Contexte 12.

$\mathrm{N}^{\circ}$ 50: Marseille. La Bourse. Contexte 9.

$\mathrm{N}^{\circ}$ 51: Marseille. Rue du Bon Jesus. Contexte 12.

$N^{\circ}$ 52-54: Marseille. La Bourse. Contexte 8.

$N^{\circ}$ 55: Rade de Marseille, découverte isolée (DRASM).

$N^{\circ}$ 56: Marseille. La Bourse. Contexte 1.

$N^{\circ}$ 57-58: Marseille. Rue du Bon-Jésus. Contexte 12.

$N^{\circ}$ 59: Marseille. La Bourse. Contexte 4.

$\mathrm{N}^{\circ}$ 60: Marseille. Rue du Bon-Jésus. contexte 12.

$N^{\circ}$ 61: Marseille. La Bourse. Contexte 4.

$N^{\circ}$ 62: Marseille. La Bourse. Contexte 1.

$\mathrm{N}^{\circ}$ 63-64: Marseille. La Bourse. Contexte 8.

$\mathrm{N}^{\circ}$ 65: Marseille. La Bourse. Contexte 7.

$N^{\circ}$ 66: Marseille. Rue du Bon-Jésus. Contexte 12.

$N^{\circ} 67$ Marseille. Cap Titol. Contexte 13.

$\mathrm{N}^{\circ}$ 68-69: Marseille. La Bourse. Contexte 8.

$\mathrm{N}^{\circ}$ 70-71: Marseille. La Bourse. Contexte 2.

$\mathrm{N}^{\circ}$ 72: Marseille. La Bourse. Contexte 10.

$N^{\circ} 73$ : Marseille. La Bourse. Contexte 1.

$\mathrm{N}^{\circ}$ 74: Marseille. La Bourse. Contexte 1.

$N^{\circ} 75$ : Marseille. rue du Bon-Jésus. Contexte 12.

No 76-77: Marseille. La Bourse. Contexte 1.

$\mathrm{N}^{\circ}$ 78-79: Marseille. La Bourse. Contexte 4.

\section{ANNEXE 2 \\ Composition des lots d'amphores}

Dans ce tableau sont indiqués, pour chaque contexte, les différents types d'amphores recensés ; le chiffre entre parenthèses correspond au nombre minimum d'individus, calculé à partir du nombre de bords augmenté du nombre excédentaire de fonds ou d'anses (divisé par deux).

\begin{tabular}{|c|l|l|l|}
\hline contextes & \multicolumn{1}{|c|}{ amphores africaines } & \multicolumn{1}{|c|}{ amphores orientales } & \multicolumn{1}{c|}{ autres amphores } \\
\hline 1 & $\begin{array}{l}\text { spatheia (13), Keay XXVII (4), Keay } \\
\text { XXXVB (8), Keay XXXVI (3), } \\
\text { indéterminées (2) }\end{array}$ & $\begin{array}{l}\text { L.R.A. 1a (29), L.R.A. 3 (26), L.R.A. } \\
\text { (a (6), L.R.A. 6 (2), Robinson M 273 } \\
(5),\end{array}$ & $\begin{array}{l}\text { Hispaniques : Dressel 23 (2), } \\
\text { Almagro 51 A-B (1) } \\
\text { Italiques : Keay LII (24) } \\
\text { Indéterminées (37) }\end{array}$ \\
\hline 2 & $\begin{array}{l}\text { spatheia (4), Keay LV (1), Keay LX } \\
\text { (4), Keay LXIIA (1), Keay LXII } \\
\text { variante (7), Bonifay 1986 Fig. 12, } \\
n^{\circ} 55 \text { (3), Keay LXIA variante (2), } \\
\text { Keay LXIC (2) }\end{array}$ & $\begin{array}{l}\text { L.R.A. 1b (5), L.R.A. 2 (3), L.R.A. 4b } \\
\text { (8), L.A. 5 (9), L.R.A. 7 (2) }\end{array}$ & Indéterminées (10) \\
\hline 3 & $\begin{array}{l}\text { Keay LXII variante (5), Bonifay } \\
\text { 1986 Fig. 12, n 55 (4), Keay LXIA } \\
\text { (2), Keay LXI variante (1) }\end{array}$ & $\begin{array}{l}\text { L.R.A. 1b (1), L.R.A. 2 (1), L.R.A. 4 } \\
\text { (1), L.R.A. 5 (1) }\end{array}$ & Indéterminée (1) \\
\hline 4 & $\begin{array}{l}\text { Keay XXV-2 (1), spatheia (2), Keay } \\
\text { XXXVB (1), Albenga 11-12 (1 ?) }\end{array}$ & $\begin{array}{l}\text { L.R.A. 1a (4), L.R.A. 3 (2), L.R.A. 4a } \\
\text { (2), Robinson M 273 (1) }\end{array}$ & $\begin{array}{l}\text { Hispaniques : Dressel 23 (1), } \\
\text { Almagro 51 A-B (1) } \\
\text { Italiques : Keay LII (3) } \\
\text { Indéterminées (3) }\end{array}$ \\
\hline
\end{tabular}


ANNEXE 2

Composition des lots d'amphores

\begin{tabular}{|c|c|c|c|}
\hline 5 & $\begin{array}{l}\text { Africaine IID (1), Keay XXV-2 (1), } \\
\text { spatheion (1), Keay XXXIII (1 ?), } \\
\text { Keay LV (1), Keay LVII (1 ?), } \\
\text { indéterminées (7) }\end{array}$ & $\begin{array}{l}\text { L.R.A. 1a (8), L.R.A. } 3 \text { (1), L.R.A. 4a } \\
\text { (1), L.R.A. } 5 \text { (1), L.R.A. } 6 \text { (1) }\end{array}$ & $\begin{array}{l}\text { Hispaniques : Almagro } 51 \text { A-B (1) } \\
\text { Italiques : Keay LII (3) } \\
\text { Indéterminées (9) }\end{array}$ \\
\hline 6 & $\begin{array}{l}\text { spatheia (5), Keay VIIIB (4), Keay } \\
\text { XXXV (1), Keay LV (4), Keay LVII } \\
\text { (2), Keay LXII (11), indéterminées } \\
(7)\end{array}$ & $\begin{array}{l}\text { L.R.A. 1a (6), L.R.A. } 2 \text { (1), L.R.A. } 3 \\
\text { (1), L.R.A. 4a (4), L.R.A. } 5 \text { (1) }\end{array}$ & $\begin{array}{l}\text { Italiques : Keay LII (2) } \\
\text { Indéterminées (9) }\end{array}$ \\
\hline 7 & $\begin{array}{l}\text { petits spatheia (2), Keay VIIIB (1), } \\
\text { Keay LXII variante (13), } \\
\text { indéterminées (7) }\end{array}$ & $\begin{array}{l}\text { L.R.A. 1b (9), L.R.A. } 2 \text { (4), L.R.A. } 3 \\
\text { (1), L.R.A. 4b (3), L.R.A. } 5 \text { (6), } \\
\text { L.R.A. } 6 \text { (1), L.R.A. } 7 \text { (1) }\end{array}$ & $\begin{array}{l}\text { Italiques: Keay LII (1) } \\
\text { Indéterminées (9) }\end{array}$ \\
\hline 8 & $\begin{array}{l}\text { spatheion (1), Keay LXII variante } \\
\text { (31), Bonifay 1986 Fig. 12, n } 55 \text { (1), } \\
\text { Keay LXIC (6), indéterminées (7) }\end{array}$ & $\begin{array}{l}\text { L.R.A. } 1 \text { b (7), L.R.A. } 2 \text { ( 5), L.R.A. } 3 \\
\text { (1), L.R.A. } 4 \text { b (15), L.R.A. } 5 \text { (12) }\end{array}$ & Indéterminées (8) \\
\hline 9 & $\begin{array}{l}\text { Africaine IID (1), Keay XXV-2 (1), } \\
\text { spatheia (12), Keay XXXVA (3), } \\
\text { Keay XXXVB (7), Keay XXXVI (4), } \\
\text { Albenga 11-12 (2), indéterminées } \\
(14)\end{array}$ & $\begin{array}{l}\text { L.R.A. 1a (26), L.R.A. } 3 \text { (1), L.R.A. } \\
4 \mathrm{a}(1)\end{array}$ & $\begin{array}{l}\text { Hispaniques : Dressel } 23 \text { (1), } \\
\text { Almagro 51 A-B (1) } \\
\text { Italiques : Keay LII (3) } \\
\text { Indéterminées (17) }\end{array}$ \\
\hline 10 & $\begin{array}{l}\text { spatheia (16), Keay VIIIB (1), Keay } \\
\text { XXXVA (1), Keay XXXVB ( 9), } \\
\text { Keay XXXVI (1), Albenga 11-12 (3), } \\
\text { indéterminées (7) }\end{array}$ & $\begin{array}{l}\text { L.R.A. 1a (10), L.R.A. } 3 \text { (1), L.R.A. } \\
\text { 4a (1), L.R.A. } 5 \text { (1), L.R.A. } 6 \text { (1), } \\
\text { Robinson M } 273 \text { (1) }\end{array}$ & $\begin{array}{l}\text { Italiques : Keay LII (3) } \\
\text { Indéterminées (4) }\end{array}$ \\
\hline 11 & $\begin{array}{l}\text { spatheia (2), petits spatheia (3), } \\
\text { Keay XXXVA (1), Keay XXXVB (2), } \\
\text { Keay XXXVI (1), Keay LVII (1), } \\
\text { Keay LXII (2), indéterminées (1) } \\
\end{array}$ & $\begin{array}{l}\text { L.R.A. 1a (3), L.R.A. 1b (1), L.R.A. } 2 \\
\text { (1), L.R.A. } 4 \text { (1), L.R.A. } 5 \text { (1) }\end{array}$ & Indéterminées (1). \\
\hline 12 & $\begin{array}{l}\text { Dressel } 30 \text { (1), Africaine I (2), Keay } \\
\text { XXV-2 (2), Keay XXXVB (3), Keay } \\
\text { XXXVI (1), Keay VIIIB (4), Keay } \\
\text { XC (1 ?), spatheia (11), } \\
\text { indéterminées (9) }\end{array}$ & $\begin{array}{l}\text { L.R.A. } 1 \text { (23), L.R.A. } 3 \text { (13), L.R.A. } 4 \\
\text { (6), L.R.A. } 5 \text { (1), Robinson M } 273 \\
\text { (4), Robinson M } 334 \text { (1) }\end{array}$ & $\begin{array}{l}\text { Hispaniques : Dressel } 23 \text { (3), } \\
\text { Almagro 51 A-B (1), Almagro 51C } \\
(1) \\
\text { Italiques : Keay LII (9) } \\
\text { Indéterminées (13) }\end{array}$ \\
\hline 13 & $\begin{array}{l}\text { Keay XXV-2 (1), spatheia (1), Keay } \\
\text { LXI (1), Albenga 11-12 (2), Keay } \\
\text { LX/LXI (1), indéterminées (3) }\end{array}$ & $\begin{array}{l}\text { L.R.A. 1a (2), L.R.A. } 3 \text { (1), L.R.A. } 5 \\
\text { (2) }\end{array}$ & $\begin{array}{l}\text { Italique : Keay LII (1) } \\
\text { Indéterminées (2) }\end{array}$ \\
\hline$\overline{14}$ & $\begin{array}{l}\text { Africaine IIC (1), Keay XXV (1), } \\
\text { Spatheia (4), Keay LXI (1), } \\
\text { Indéterminées (5) }\end{array}$ & $\begin{array}{l}\text { L.R.A. } 1 \text { b (8), L.R.A. } 2 \text { (1), L.R.A. } 3 \\
\text { (2), L.R.A. } 4 \text { (1), L.R.A. 4b (1), } \\
\text { L.R.A. } 5 \text { (1), L.R.A. } 7 \text { (1), Robinson } \\
\text { M } 273 \text { (1) }\end{array}$ & $\begin{array}{l}\text { Hispaniques : Almagro } 50 \text { (1), } \\
\text { Almagro 51 A-B (1) } \\
\text { Indéterminée (1) }\end{array}$ \\
\hline 15 & Keay LXI (1), indéterminée (1) & $\begin{array}{l}\text { L.R.A. 1B (1), L.R.A. 2a (1), L.R.A. } \\
\text { 4b (1), L.R.A. } 5 \text { (1), Unguentarium } \\
\text { tardif (1) }\end{array}$ & Indéterminée (1) \\
\hline 16 & Keay LXIC (1), indéterminée (1) & $\begin{array}{llll}\begin{array}{l}\text { L.R.A.(1), L.R.A. } \\
\text { indéterminée (1) }\end{array} & 4 & (1), \\
\end{array}$ & Indéterminée (1) \\
\hline 17 & $\begin{array}{l}\text { Keay LXI-LXII (1), Keay VIIIA (2), } \\
\text { Keay L (2) }\end{array}$ & $\begin{array}{lllll}\text { L.R.A. } 1 \text { (1), } & \text { L.R.A. } & 4 & (1), \\
\text { indéterminée (1) }\end{array}$ & $\begin{array}{l}\text { Amphore à corps globulaire (1) } \\
\text { Indéterminée (1) } \\
\end{array}$ \\
\hline 18 & $\begin{array}{l}\text { Keay XXV-2 (1), spatheion tardif (1), } \\
\text { Keay XXXVB (1), Keay XXXVI (2), } \\
\text { Keay VIIIA (1), Keay LV (1), Keay } \\
\text { LXII (2), Keay LXI-LXII (2), } \\
\end{array}$ & $\begin{array}{l}\text { L.R.A. } 1 \text { (1), L.R.A. } 4 \text { (1), L.R.A. } 5 \\
\text { (1), L.R.A. } 6 \text { (1) }\end{array}$ & $\begin{array}{l}\text { Hispaniques (2) } \\
\text { Indéterminées }(2)\end{array}$ \\
\hline 19 & $\begin{array}{l}\text { Spatheia tardifs (3), Keay LXI (1), } \\
\text { indéterminées (7) }\end{array}$ & $\begin{array}{l}\text { L.R.A. } 1 \text { b (3), L.R.A. } 2 \text { (1), L.R.A. 4b } \\
\text { (2), L.R.A. } 5 \text { (4), L.R.A. } 7 \text { (1), } \\
\text { indéterminée (1) }\end{array}$ & $\begin{array}{l}\text { Hispaniques (3) } \\
\text { Amphores à corps globulaire (3) } \\
\text { Indéterminées (3) }\end{array}$ \\
\hline 20 & $\begin{array}{l}\text { Keay XXV (1), Spatheion (1), } \\
\text { Spatheia tardifs (2), Keay VIIIA (1), } \\
\text { Albenga 11-12 (2), Keay LXIIA (8), } \\
\text { Keay LX (1), Keay LXIA (4), Keay } \\
\text { LXIC (3), Keay LXI-LXII (5), Keay } \\
\text { L (7), indéterminées (18) } \\
\end{array}$ & $\begin{array}{l}\text { L.R.A. 1a (3), L.R.A. 1b (8), L.R.A. } 2 \\
\text { (1), L.R.A. } 3(1), \text { L.R.A. } 4 \text { b (7), } \\
\text { L.R.A. } 5(4), \text { L.R.A. } 7 \text { (1), } \\
\text { indéterminées (4) }\end{array}$ & $\begin{array}{l}\text { Hispaniques: Dressel } 23(3) \\
\text { Italiques : Keay LII (2) } \\
\text { Amphore à corps globulaire (1) } \\
\text { Indéterminées (3) }\end{array}$ \\
\hline
\end{tabular}




\begin{tabular}{|c|c|c|c|c|c|c|c|c|c|c|c|c|c|c|c|c|c|c|c|c|}
\hline & 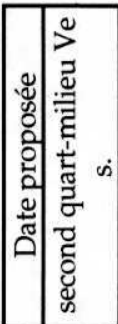 & 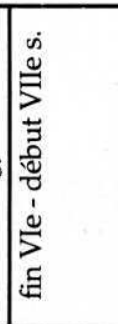 & $\stackrel{.}{3}$ & 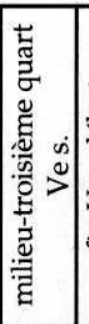 & 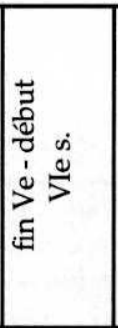 & $\stackrel{\infty}{\stackrel{\infty}{\infty}}$ & 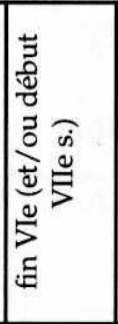 & 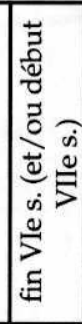 & 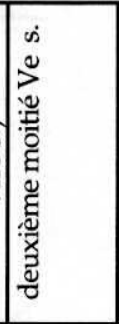 & 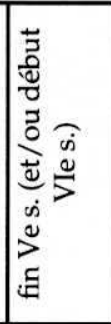 & $\stackrel{\infty}{\tilde{m}}^{\stackrel{\infty}{\nu}}$ & 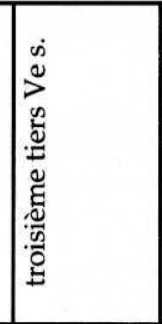 & 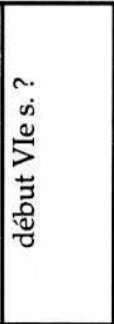 & 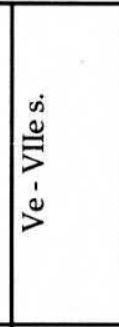 & & 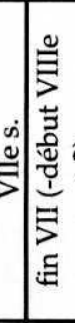 & 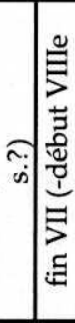 & त. &  &  \\
\hline 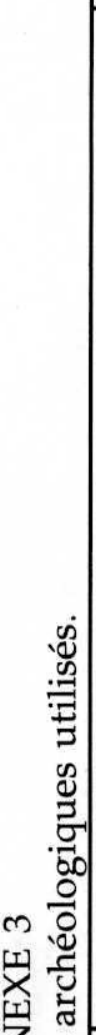 & 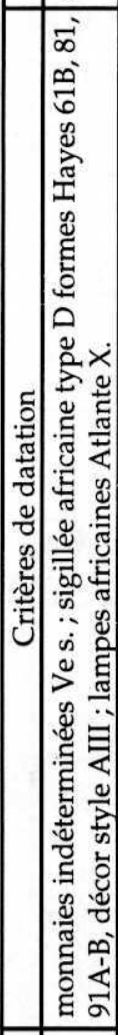 & 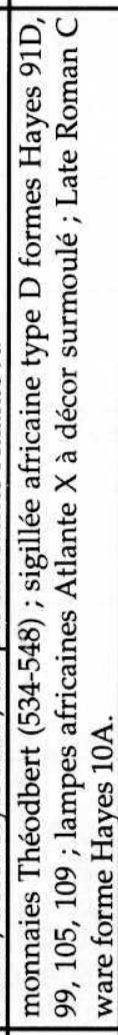 & 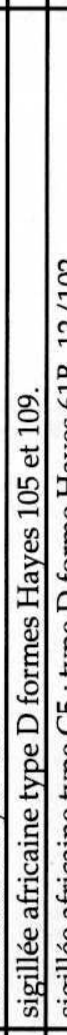 & 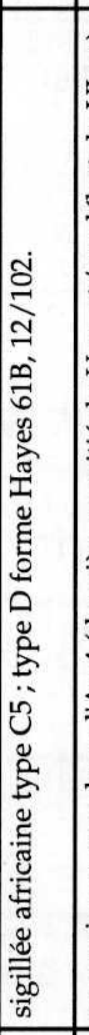 &  & 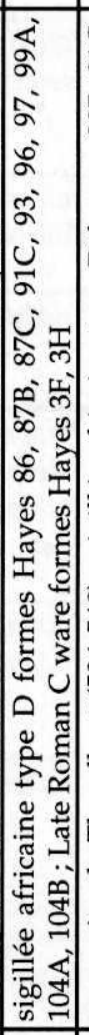 & 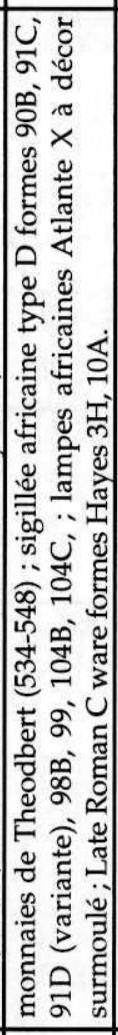 & 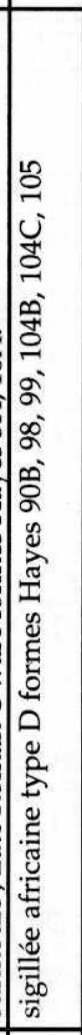 & 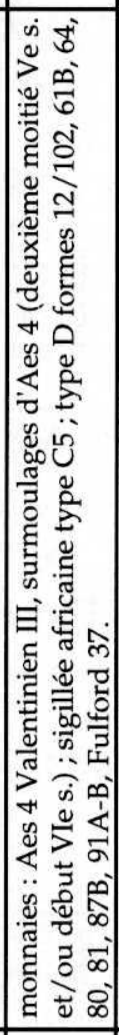 & 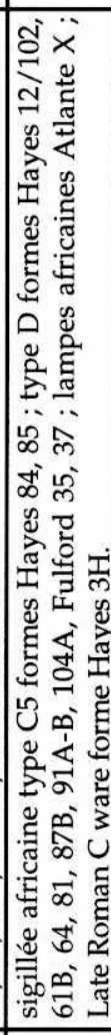 & 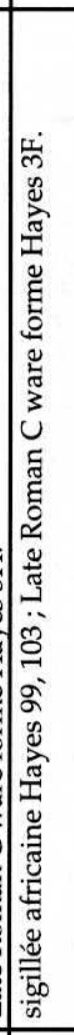 & 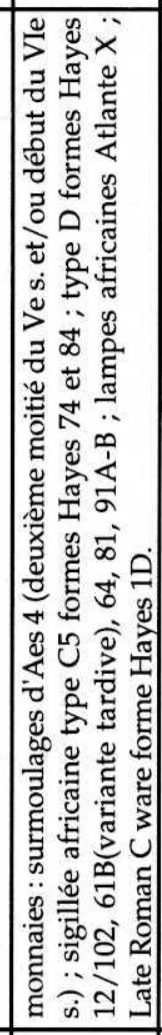 & 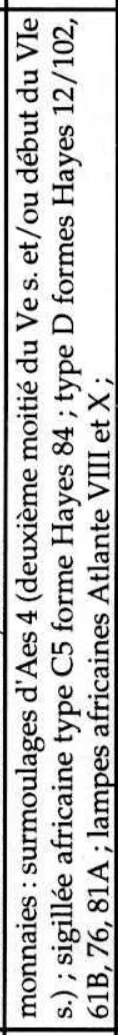 & 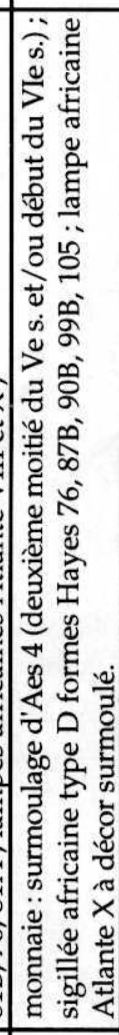 & 氛 & 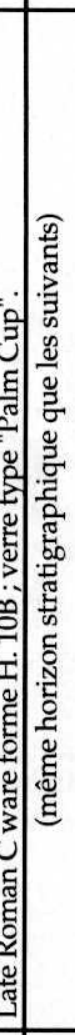 & & & 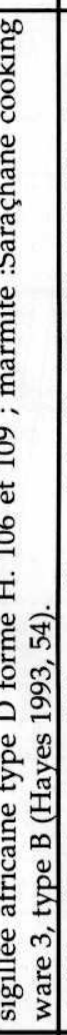 &  \\
\hline 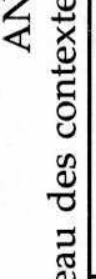 & 总 & 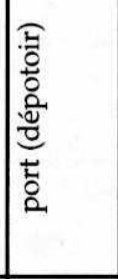 & 产 & 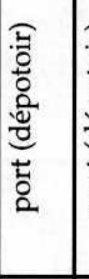 & 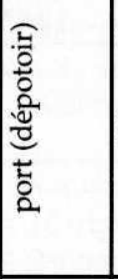 & 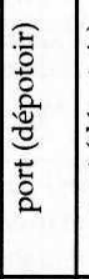 & 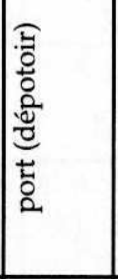 & 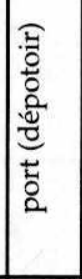 & 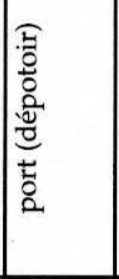 & 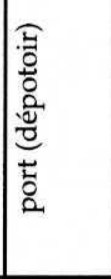 & 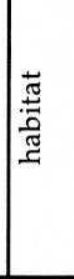 & 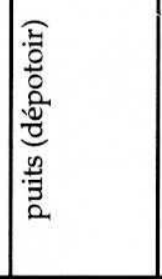 & 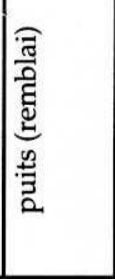 & 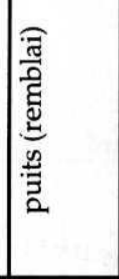 & 苞 & 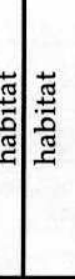 & 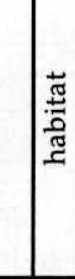 & & & 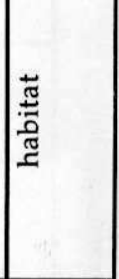 \\
\hline$\stackrel{0}{\tilde{\sigma}}$ & 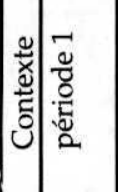 & 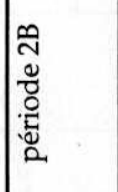 & $\left.\mid \begin{array}{c}0 \\
0 \\
\vdots \\
\vdots \\
\vdots \\
\vdots\end{array}\right]$ & 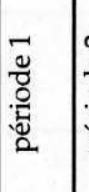 & 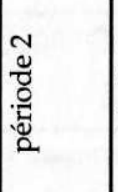 & . & 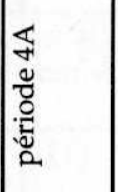 & 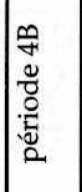 & . & 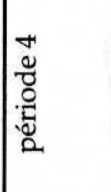 &  & 竎 & & & 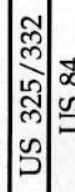 & $\begin{array}{l}\text { న్ } \\
\text { wh }\end{array}$ & $\begin{array}{l}\hat{b} \\
\text { b. }\end{array}$ & & ת & $\mid \begin{array}{l}\infty \\
2 \\
\tilde{s}\end{array}$ \\
\hline & 离害 &  & & 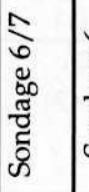 &  &  &  & 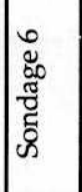 &  & 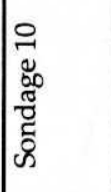 & 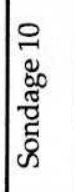 &  & 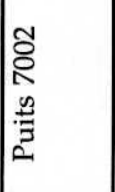 &  & $\stackrel{10}{\geq}$ & $\geq \geq$ &  & $z$ & & $\geq$ \\
\hline & 泀| & $=$ & $=$ & $=$ & $=$ & $=$ & & |= & d= & $=$ & $=$ & 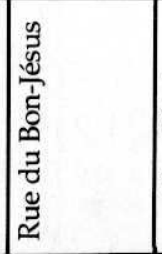 & లే & 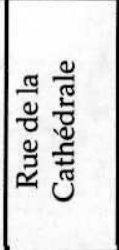 & 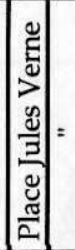 & $=$ & & & & $=$ \\
\hline & $|z|-$ & 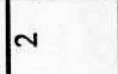 & 1 & $\theta^{+}$ & 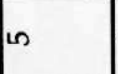 & $0^{0}$ & & $\infty$ & 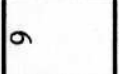 & $T$ & & T. & $\stackrel{m}{-2}$ & $F$ & & & $\Gamma$ & & & N \\
\hline
\end{tabular}

